\title{
Les carrières gallo-romaines de Rezé (Loire-
} Atlantique)

The Gallo-Roman quarries of Rezé (Loire-Atlantique)

Alexandre Polinski et Lionel Pirault

\section{OpenEdition}

\section{Journals}

Édition électronique

URL : https://journals.openedition.org/rao/1844

DOI : $10.4000 /$ rao. 1844

ISBN : 978-2-7535-2790-4

ISSN : $1775-3732$

\section{Éditeur}

Presses universitaires de Rennes

\section{Édition imprimée}

Date de publication : 30 décembre 2012

Pagination : 201-222

ISBN : $978-2-7535-2641-9$

ISSN : 0767-709X

\section{Référence électronique}

Alexandre Polinski et Lionel Pirault, « Les carrières gallo-romaines de Rezé (Loire-Atlantique) », Revue archéologique de l'Ouest [En ligne], 29 | 2012, mis en ligne le 30 décembre 2014, consulté le 19 août 2022. URL : http://journals.openedition.org/rao/1844 ; DOI : https://doi.org/10.4000/rao.1844

Ce document a été généré automatiquement le 19 août 2022.

Tous droits réservés 


\title{
Les carrières gallo-romaines de Rezé (Loire-Atlantique)
}

The Gallo-Roman quarries of Rezé (Loire-Atlantique)

\author{
Alexandre Polinski et Lionel Pirault
}

\section{NOTE DE L'ÉDITEUR}

Manuscrit reçu le 11 juillet 2011, accepté le 19 novembre 2011.

Les exemples de carrières dont l'Antiquité est avérée par la fouille sont relativement peu nombreux à l'échelle du Grand-Ouest de la France ${ }^{1}$. On ne peut guère citer que la carrière de granite de Locuon à Ploërdut dans le Morbihan, qui a fourni une grande partie du décor architectonique de Carhaix, chef-lieu des Osismes (Eveillard et al., 1997 ; Maligorne et al., 2002), et se rapproche par sa superficie et les procédés d'extraction mis en œuvre des grandes carrières de calcaire ou de grès décrites dans le Sud et l'Est de la France (Bessac et Sablayrolles, 2002). Il existait pourtant, à côté d'un petit nombre de vastes carrières destinées à alimenter en pierre de taille les centres urbains ou ruraux, une multitude de sites d'extraction de taille modeste, dont la fonction se limitait exclusivement à fournir la pierre nécessaire à l'empierrement ou aux maçonneries, soit une part essentielle des besoins en pierres d'une cité. Quelques excavations sont parfois signalées dans les rapports finaux d'opération archéologique ${ }^{2}$, mais un certain nombre échappe probablement à l'attention de l'archéologue, n'est pas identifié comme des carrières ou est l'objet d'un certain désintérêt. Un simple regard sur la littérature portant sur la thématique de la pierre permet en tout cas de constater qu'elles ne sont pratiquement jamais publiées, les carrières de l'île Lavret et de la Boisanne à Plouërsur-Rance, dans les Côtes-d'Armor, faisant figure d'exceptions (Giot, 1996, p.17; Menez, 1996, p. 88-89, 194, fig.). Il semblait donc intéressant de présenter un ensemble de petits sites d'extraction mis au jour à Rezé, en Loire-Atlantique, d'autant plus que l'extraction concerne ici pour l'essentiel des roches métamorphiques, matériaux généralement peu abordés dans la problématique des carrières antiques. Si le terme de 
«site d'extraction » paraît plus juste en raison de la faible superficie ou profondeur de la plupart des excavations, celui de "carrière" sera tout de même utilisé par commodité.

\section{Contexte géographique et archéologique}

2 La ville antique qui se développe à Rezé (Ratiatum) est située en fond d'estuaire, un peu en aval de Nantes et sur la rive gauche de la Loire, sur un territoire généralement attribué aux Pictons (fig. 1). Créée ex-nihilo pendant la période augustéenne, sur un coteau au relief peu accentué et en bordure d'un ancien bras de Loire aujourd'hui comblé, cette agglomération secondaire connaît dès le $\mathrm{I}^{\text {er }}$ siècle apr. J.-C. un développement rapide, sans doute lié à une activité portuaire prospère, et atteint son apogée dans le courant du II ${ }^{\mathrm{e}}$ siècle (Deschamps et al., 1992 ; Deschamps, Pirault, 1999 ; Pirault, 2001 ; 2004 ; Pirault, Guitton, 2001). Elle occupe alors une superficie de 40 à 50 hectares, si on ne tient compte que des espaces réellement urbanisés, et s'étend sur au moins un kilomètre de longueur et 300 à 400 mètres de largeur, entre le quartier des Couëts (commune de Bouguenais) à l'ouest et le quartier Saint-Lupien à l'est (fig. 2). Le $\mathrm{III}^{\mathrm{e}}$ siècle marque une période de récession, à mettre probablement en relation avec un envasement progressif du port, mais les activités se poursuivent tout au long de l'Antiquité tardive dans plusieurs secteurs.

Figure 1 : Localisation de l'agglomération antique de Rezé. DAO L. Pirault. Figure 1: Location of the ancient small town of Rezé.

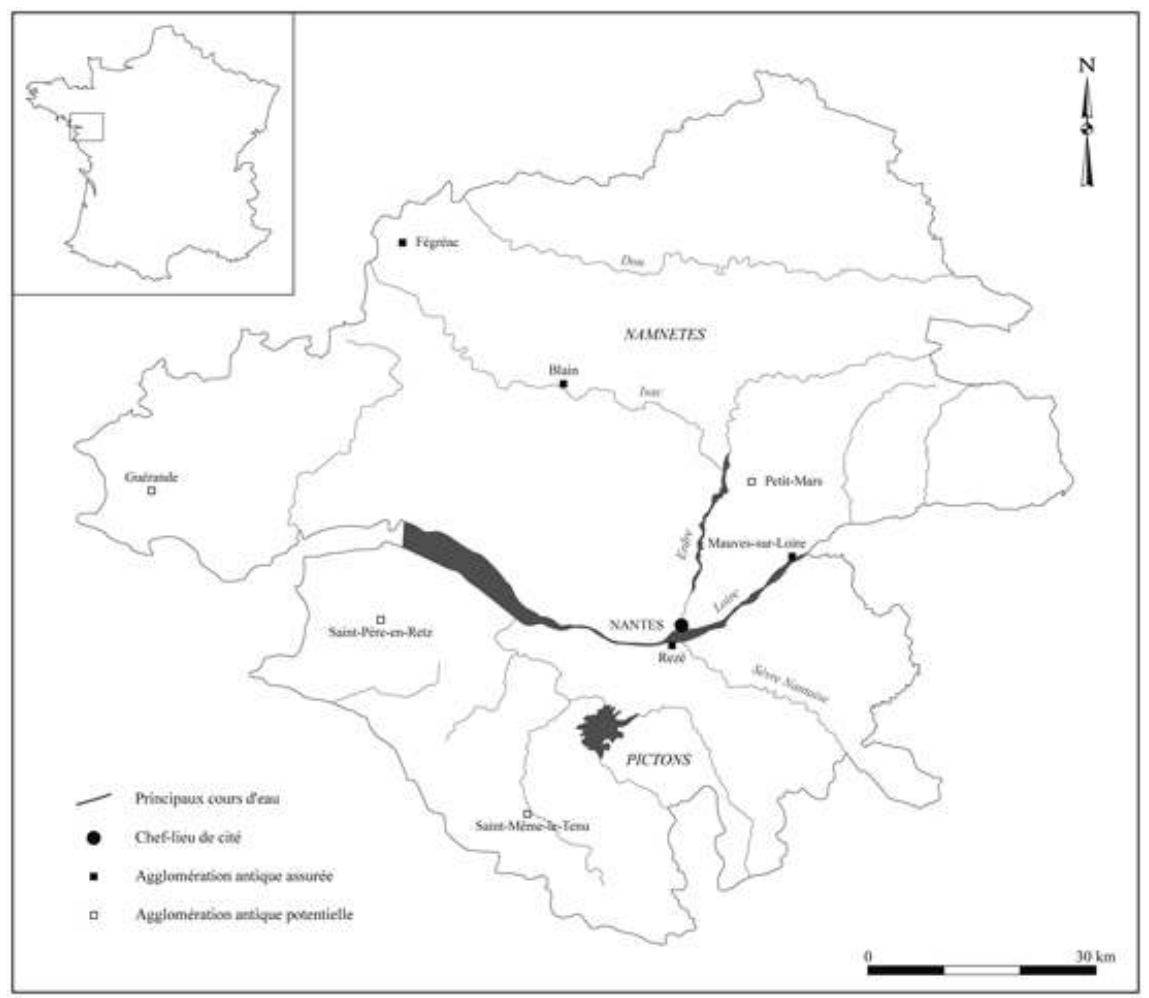


Figure 2 : Plan de l'agglomération antique de Rezé au ॥e apr. J.-C. avec la localisation des quartiers de la Bourderie et de Saint-Lupien. DAO L. Pirault, M. Monteil.

Figure 2: Plan of the ancient small town of Rezé in the $2^{\text {nd }}$ century AD with location of La Bourderie and Saint-Lupien districts.

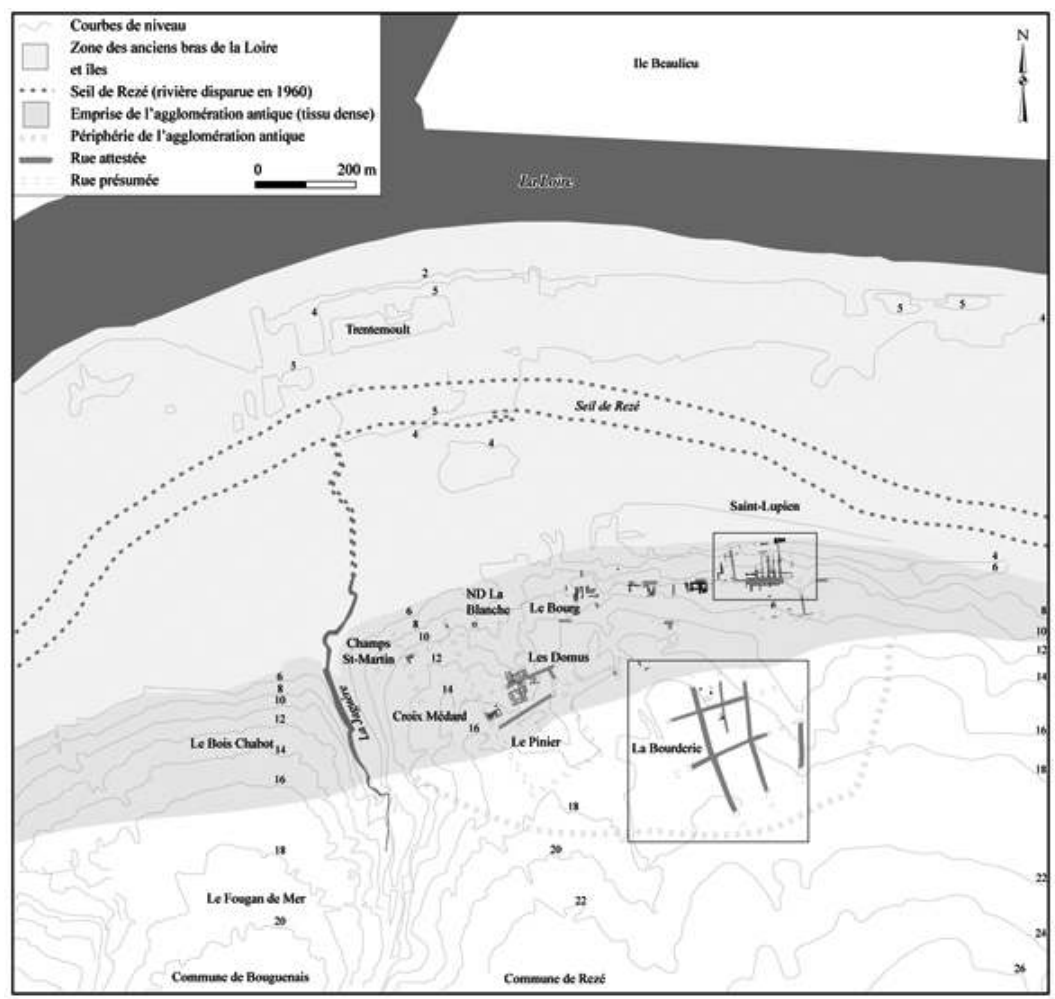

3 Les sites d'extraction faisant l'objet du présent article se concentrent dans la partie orientale de la ville et se répartissent entre les quartiers dits de Saint-Lupien et de la Bourderie. Le premier, à vocation artisanale, commerciale et portuaire, est caractérisé au II $^{\mathrm{e}}$ siècle apr. J.-C. par un ensemble de boutiques et d'entrepôts, ainsi que par des aménagements portuaires et des thermes publics, après avoir subi toute une série de réaménagements successifs dans le courant du $\mathrm{i}^{\mathrm{er}}$ siècle (Arthuis et al., 2007 ; 2010a ; $2010 \mathrm{~b}$; Peretti, 2010). Le second, situé au sud de Saint-Lupien et occupant une position périphérique, connaît un phénomène de pré-urbanisation durant la période tibérienne, avec la mise en place d'un réseau viaire délimitant un ensemble d'îlots, mais il ne restera que partiellement urbanisé et ne sera guère occupé que par un atelier de potier, un atelier de verrier et une nécropole à incinération (Pirault et al., 2001 ; Pirault, Bellanger, 2008).

\section{Cadre géologique}

D'un point de vue géologique, l'agglomération antique de Rezé est installée sur un terrain métamorphique formé d'une alternance de micaschistes et de gneiss orientés nord-ouest/sud-est ${ }^{3}$. Les micaschistes, dont les lits ne dépassent pas un demimillimètre d'épaisseur, sont finement foliés et à deux micas, avec quelques petites lentilles millimétriques de feldspath. Les gneiss, dont les lits ne dépassent pas un millimètre d'épaisseur, sont eux aussi finement foliés et à deux micas, avec des grains de quartz et de feldspath millimétriques. La muscovite et la biotite y sont particulièrement abondantes. 
5 Sur le site de Saint-Lupien, un examen attentif du substrat a permis de constater l'existence de quelques bandes de granite pegmatitique de puissances variables, non signalées par la carte géologique, et dont l'orientation oscille entre Nord $70^{\circ}$ et Nord $130^{\circ}$. Ce granite est caractérisé par des cristaux plurimillimétriques à pluricentimétriques de feldspath bien blanc, de quartz et de muscovite et montre parfois de petits cristaux noirs de tourmaline en amas peu épais et pluricentimétriques. Trois bandes d'amphibolite, orientées Nord $130^{\circ}$ et Nord $150^{\circ}$, ont également été entrevues au sud de la chapelle Saint-Lupien et montrent une roche finement foliée de couleur vert sombre, dont les lits ne dépassent pas un demi-millimètre d'épaisseur et qui est constituée de cristaux millimétriques de hornblende verte et de quelques lentilles millimétriques de feldspath plagioclase. Le site de la Bourderie a en outre permis d'observer localement quelques bandes de gneiss leptynitique cisaillé, orientées à environ Nord $75^{\circ}$. Ce gneiss, dont les lits ne dépassent pas un millimètre d'épaisseur, est finement folié et montre des cristaux millimétriques de quartz et de feldspath plus ou moins étirés et une biotite déformée en amas.

6 Toutes ces roches constituant le substrat local sont fortement diaclasées, c'est-à-dire recoupées par un réseau de fissures horizontales, verticales ou obliques. Cette fracturation, plus ou moins dense selon les secteurs, délimite naturellement des fragments de dimensions variables, allant du petit moellon au bloc de plusieurs dizaines de centimètres de largeur.

\section{Présentation des sites d'extraction}

\section{Les carrières de la Bourderie}

7 Les terrains de la Bourderie sont situés à 300 mètres au sud-est du centre bourg historique de Rezé (paroisse Saint-Pierre). Ils couvrent une surface de 51884 mètres carrés, comprise entre la rue Théodore-Brosseau à l'ouest, la rue Victor-Hugo à l'est et la rue Georges-Boutin au nord. Au sud, ils sont limités par la voie SNCF de Nantes à Pornic (fig. 3). 
Figure 3 : Plan du quartier de la Bourderie avec la localisation des sites d'extraction. DAO L. Pirault. Figure 3: Plan of La Bourderie district with location of quarries.

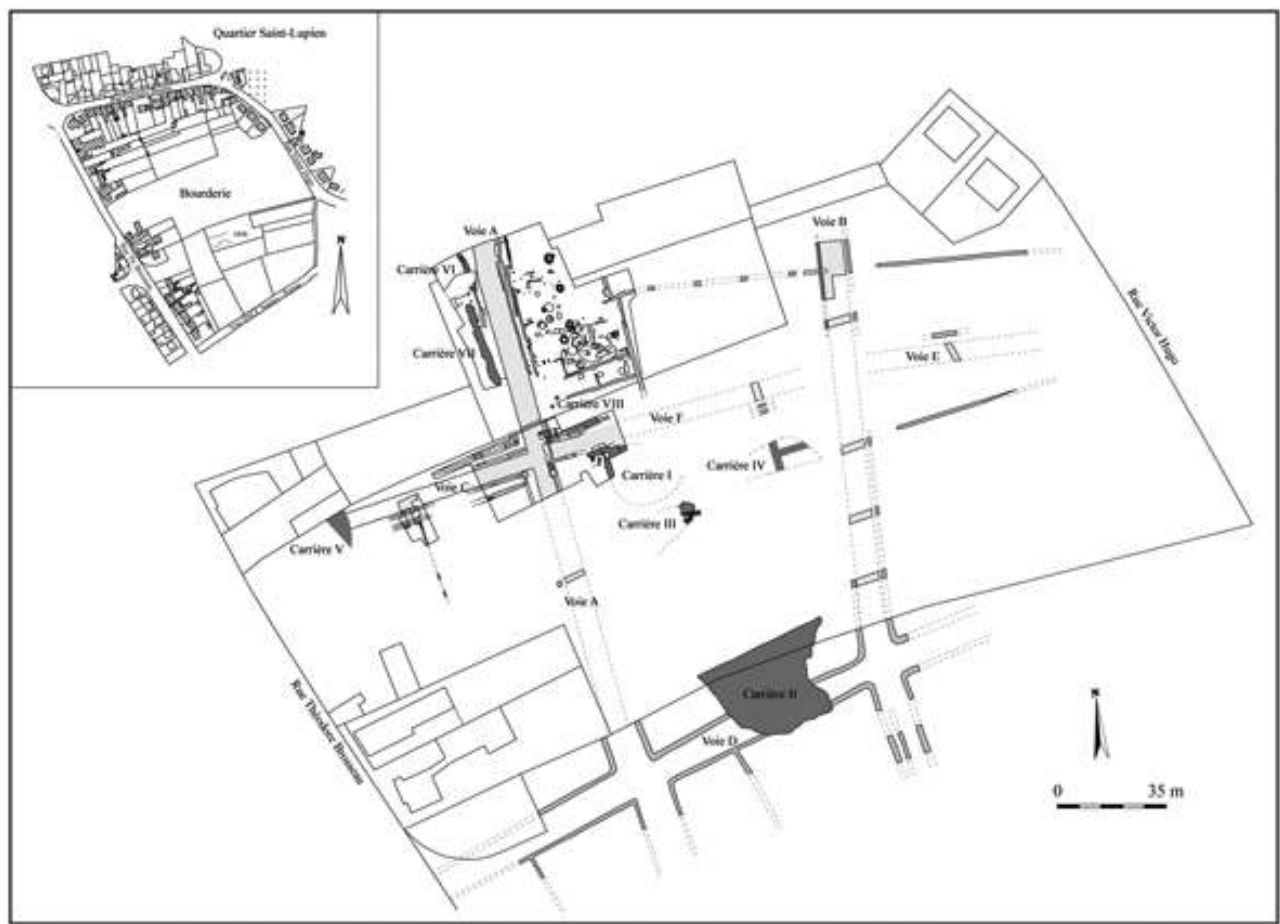

Les différentes campagnes de sondages et de fouilles programmées réalisées entre 1996 et 2000 dans l'emprise de l'ancienne tenue maraîchère de la Bourderie ont mis en évidence un réseau de rues d'époque gallo-romaine, construit dans le courant du $\mathrm{I}^{\mathrm{er}}$ siècle de notre ère et délimitant des îlots partiellement vides de toute occupation (Pirault, $1996 ; 1997 ; 1998 ; 1999 ; 2000 ; 2003)^{4}$.

Elles ont également révélé l'existence d'une exploitation de la pierre dont les premières extractions ont débuté à l'époque romaine et qui, pour certaines, ont perduré jusqu'au $\mathrm{XIX}^{\mathrm{e}}$ siècle. Ces carrières de types différents, étendues et de forme longiligne, ont en commun, à une exception près, d'être implantées sur la même bande de gneiss massif, dont l'émergence a été mise en saillie par l'érosion différentielle du socle.

\section{Les sites d'extraction étendus : carrières I, II, III, IV et V}

Ces carrières au nombre de cinq s'étendent sur des superficies importantes (fig. 3). Si l'origine antique de la plupart d'entre elles ne fait aucun doute, on ignore le détail de la chronologie de leurs séquences d'exploitation jusqu'au terme de leur usage au $\mathrm{XIX}^{\mathrm{e}}$ siècle.

Dans la partie centrale de l'ancienne tenue maraîchère, la topographie du terrain est marquée par la présence d'un puisard agricole isolé et partiellement comblé. Ce dernier a permis de localiser un point d'extraction de la roche (carrière I) que l'on suppose encore en usage à une période peu ancienne. Les sondages pratiqués autour de cet espace ont montré l'existence de deux autres zones d'extraction. L'une, située au sudest (carrière IV), semble peu étendue mais relativement profonde (environ $2,50 \mathrm{~m}$ de profondeur) et l'autre (carrière III) jouxte la limite méridionale de la carrière I. La carrière II est située plus au sud. On ne connaît son étendue que de manière approximative et sa profondeur moyenne, au niveau du front de taille, a été obtenue 
par sondages ponctuels (Pirault, 1997). Enfin, la cinquième zone d'extraction (carrière V), qui n'a pas fait l'objet de sondage de reconnaissance, est localisée dans la partie ouest du terrain. Elle se prolonge en dehors de la limite de fouille vers la rue Théodore-Brosseau.

\section{Carrière I}

L'emprise exacte de la carrière I reste approximative. Le puisard agricole qui la recoupe permet d'en délimiter l'extension orientale et une fenêtre de sondage sa limite méridionale. À l'ouest, une portion relativement limitée a fait l'objet d'une fouille exhaustive (fig. 3, 4, 5 et 6). À cet emplacement, on remarque un creusement en forme d'appendice situé parallèlement au sud d'une section de voie antique assez arasée (voie F). Ce creusement d'une longueur de 10 mètres, qui se prolonge à l'est vers la zone d'extraction, offre une forte déclivité de 1,65 mètre d'ouest en est. Ce dernier pourrait correspondre à un accès vers la zone d'exploitation.

Figure 4 : Front de taille nord de la carrière I. Cliché L. Pirault. Figure 4: North secondary quarry face of quarry $\mathrm{l}$.

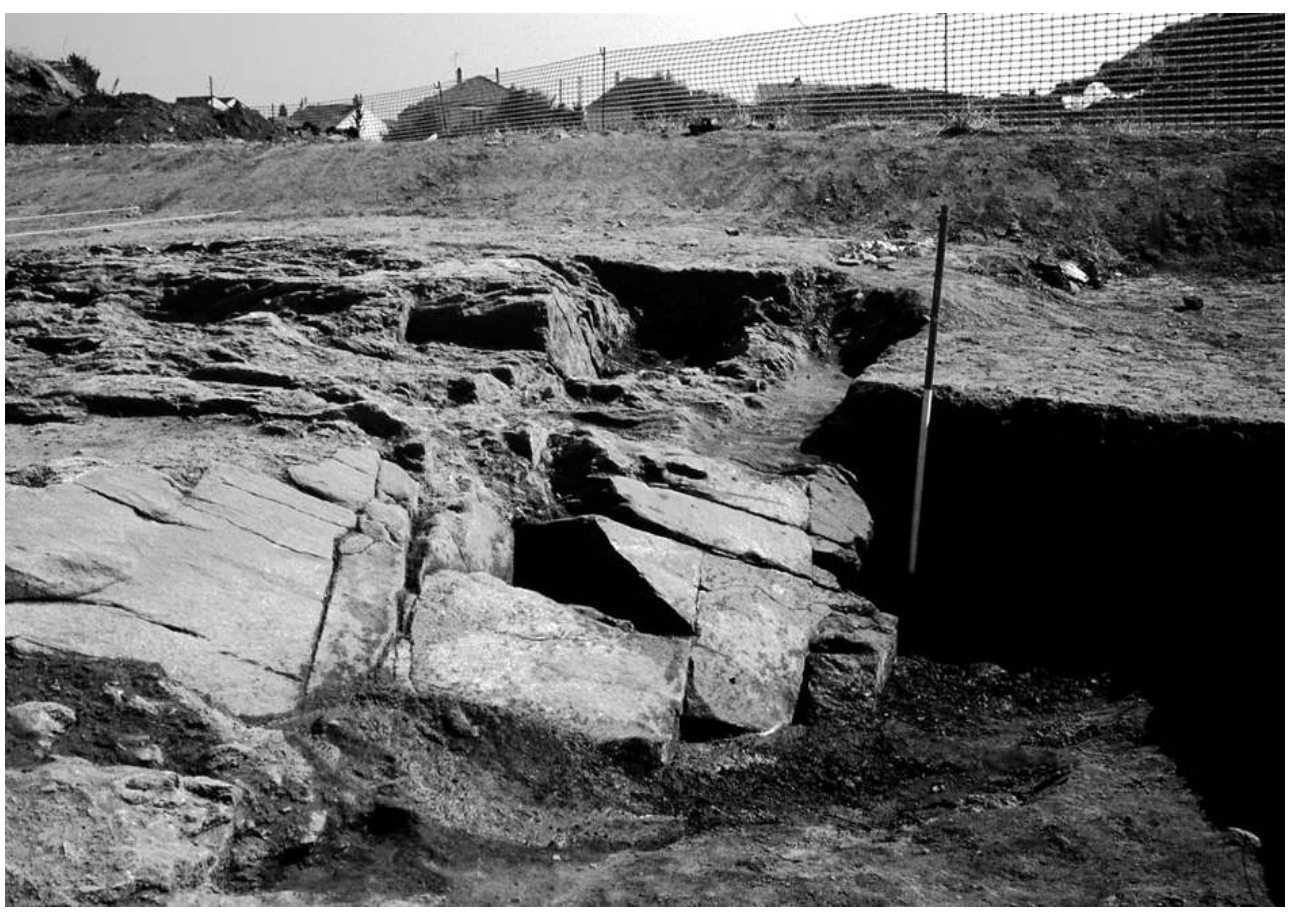


Figure 5 : Carrière I vue de l'Ouest. Cliché L. Pirault. Figure 5: Quarry I taken from west.

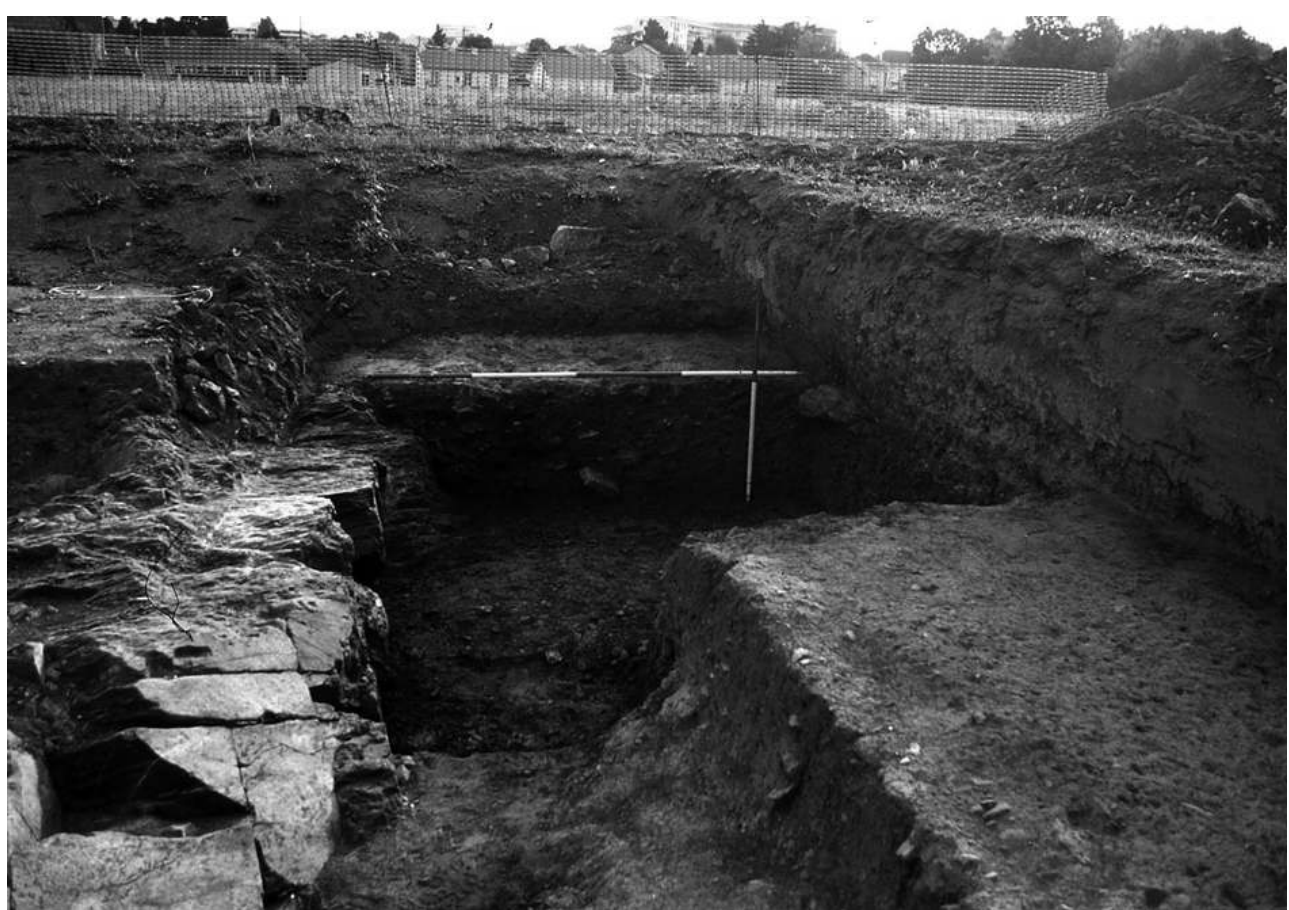

Figure 6 : Coupes et implantation des coupes de la carrière I. DAO L. Pirault. Figure 6: Sections of quarry I and their location.

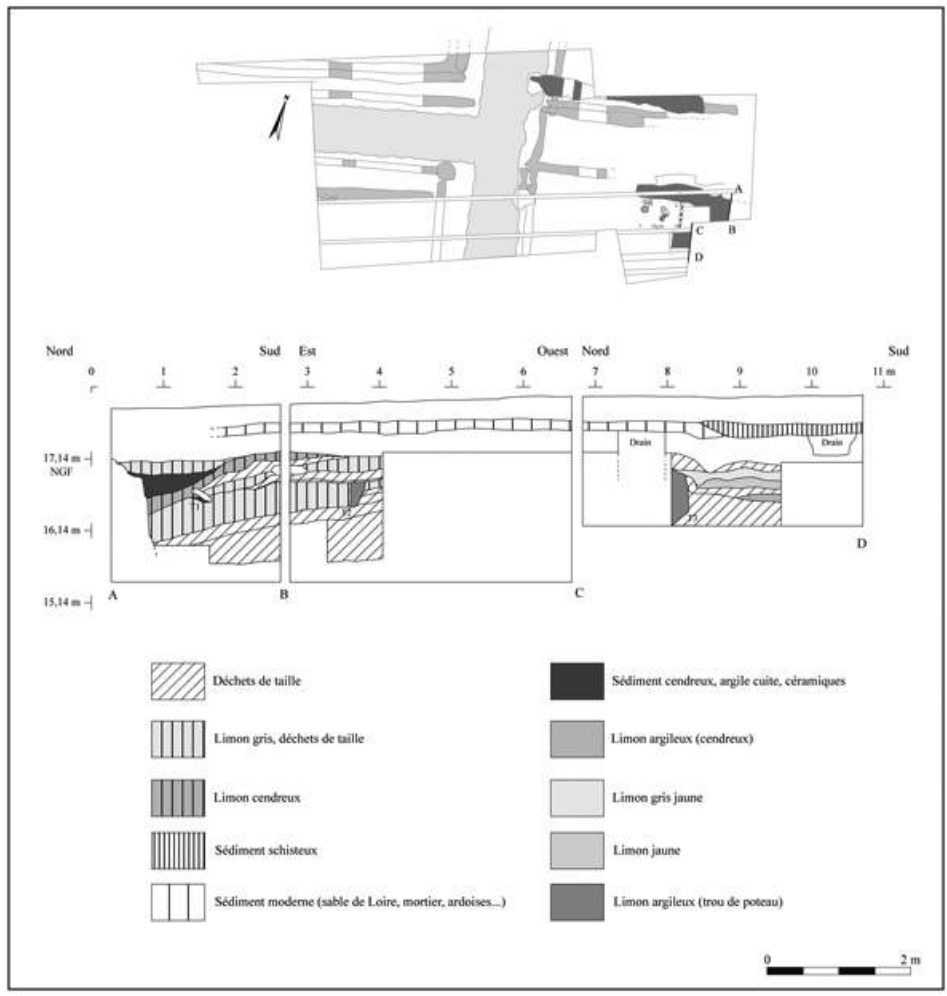

La fouille a montré que le comblement, d'une puissance moyenne de 1,60 mètre, était essentiellement constitué par des déchets de taille formant des lits d'épaisseur irrégulière qui reposaient sur le substrat nivelé à l'altitude de 15,70 mètres NGF. Il a été 
remarqué que certains dépôts interstratifiés étaient composés par des sédiments limoneux plus ou moins épais qui pourraient être liés à des reprises temporaires de la sédimentation, ou du moins à des périodes de stagnation en milieu ouvert. De même, l'examen de la stratigraphie a révélé la présence de plusieurs trous de poteaux de différents diamètres étagés dans le remblaiement. Si leur fonction demeure difficile à définir clairement, on peut toutefois supposer qu'ils devaient contribuer à maintenir et stabiliser les déchets de taille en dehors du périmètre d'extraction.

Près de la bordure nord de la carrière, parallèlement au plan de débitage, un dépôt cendreux d'une trentaine de centimètres d'épaisseur, contenant une très forte densité de déchets liés à un atelier de verrier voisin (cendres, fragments de fours, verres concassés, groisils...), comble la dépression occasionnée par le tassement des matériaux sous-jacents. Ces éléments caractéristiques, datables de la fin du $\mathrm{I}^{\mathrm{er}}$ siècle de notre ère, scellent seulement une partie relativement restreinte du comblement de la carrière I. Cet enchaînement stratigraphique assuré et clairement identifié permet d'affirmer que cette zone d'exploitation de la pierre a été abandonnée dans les dernières décennies du $\mathrm{I}^{\text {er }}$ siècle apr. J.-C.

Les limites nord et est, qui coupent l'emprise de l'axe de circulation F, correspondent quant à elles à l'utilisation tardive de la carrière, dont l'usage a perduré jusqu'à nos jours comme mare agricole.

\section{Carrière II}

Contrairement à la carrière I, il ne subsiste aucune trace apparente permettant de localiser la carrière II. Son emplacement approximatif est toutefois indiqué sur le cadastre moderne $^{5}$, sous la forme d'une limite en pointillés, mais cette partie des terrains apparaît aujourd'hui parfaitement nivelée. Un front de taille d'une longueur totale de 44 mètres limite la zone d'extraction au nord et un décapage d'une surface de 320 mètres carrés a permis d'en connaître la limite orientale (fig. 3). Dans cette partie du site, une masse considérable de déchets de taille de tout calibre occupe l'intégralité des surfaces ouvertes. Dans la partie est, les matériaux qui constituent le comblement superficiel sont peu compactés et sont mêlés à du mobilier moderne (XIX siècle). À ce même emplacement, la voie $\mathrm{D}$ a été totalement détruite par l'extension méridionale de la zone d'extraction.

Un sondage d'une quinzaine de mètres de longueur, réalisé au nord parallèlement au front de taille, montre que ce dernier atteint une hauteur de 4,50 mètres. La base du comblement est occupée sur une épaisseur de 0,50 mètre par un sédiment limoneux de couleur grise recélant une quantité considérable de mobilier attribuable à la fin du $\mathrm{II}^{\mathrm{e}}$ siècle de notre ère. Ce véritable dépotoir urbain était recouvert par une couche de matériaux de démolition peu fragmentés (tegulae, imbrices, moellons calibrés, blocs de calcaire...) d'une épaisseur de près d'un mètre.

\section{Carrière III}

L'extension vers l'ouest de la carrière III n'est pas connue (fig. 3). Les sondages n'ont permis d'en délimiter la surface qu'à l'est sur une portion relativement réduite. Un sondage réalisé dans la cavité le long du front de taille a permis d'atteindre le fond à une profondeur de 2,50 mètres. À la base, un comblement limoneux chargé de charbons de bois, d'une trentaine de centimètres d'épaisseur, a livré un abondant mobilier 
datable du milieu du II ${ }^{e}$ siècle de notre ère. Ce dépôt était scellé sous une épaisse masse de matériaux constituée exclusivement par des déchets de taille décimétriques. Dans la partie sommitale, un petit bâtiment gallo-romain composé d'au moins deux pièces aux dimensions exiguës était directement construit sur les matériaux de comblement (Pirault, 1996). Le mobilier recueilli sur les sols d'occupation de cette construction indique qu'elle a été abandonnée dans les premières décennies du ${ }^{\mathrm{e}}{ }^{\mathrm{e}}$ siècle de notre ère.

\section{Carrière IV}

19 La superficie de la carrière IV demeure imprécise. Deux sondages perpendiculaires permettent seulement d'appréhender globalement des limites qui laissent à penser qu'il s'agit d'un point d'extraction dont la surface était relativement réduite. Au niveau des sondages, le fond de la carrière est situé à une profondeur de 2,50 mètres (fig. 3). La présence de la nappe phréatique ou d'une source n'a pas permis d'explorer plus précisément cette zone d'extraction. Le comblement était exclusivement constitué de déchets de taille fortement compactés.

\section{Carrière V}

20 À l'ouest des terrains de la Bourderie, le tracé de la voie antique $C$ est coupé par une carrière dont on ne connaît qu'une limite située au niveau de l'axe de circulation (fig. 3). Elle s'étend sans aucun doute à l'ouest vers la rue Théodore-Brosseau, où l'on remarque encore dans le parc de la Maison Radieuse de vastes cavités remplies d'eau qui correspondent à des carrières modernes encore en usage au début du $\mathrm{xx}^{\mathrm{e}}$ siècle.

\section{Les carrières longilignes : carrières VI, VII et VIII}

21 Ces zones d'extraction au nombre de trois ont une situation topographique et une configuration analogues. En effet, de formes allongées, elles jouxtent invariablement un axe de circulation (voie A), situé dans la partie nord du terrain et qui constitue l'axe principal d'accès à l'agglomération antique (fig. 3 et 7). Son tracé est identifiable sur une longue distance au sud de la commune de Rezé et son prolongement vers Poitiers a été reconnu en divers points au-delà des limites communales.

\section{Carrière VI}

La carrière VI est située en limite de fouille, sur la bordure ouest de la voie A. Il s'agit d'un creusement linéaire d'une largeur de 1,60 mètre pour une profondeur moyenne de 0,50 mètre, qui a été dégagé sur une longueur de 12 mètres (fig. 7). Au sud, sur un tiers de sa longueur, il apparaît perturbé par une carrière assez récente (xıx ${ }^{e}$ siècle). Ce site d'extraction, dont le prolongement au nord n'est pas connu, présente la particularité d'être ouvert dans des micaschistes altérés impropres à toute construction et de recouper une bande de gneiss leptynitique cisaillé (A) large de deux mètres et orientée à environ Nord $75^{\circ}$. 
Figure 7 : Nature du substrat dans lequel sont ouvertes les carrières I, VI, VII et VIII. DAO L. Pirault. Figure 7: Nature of the substratum in which the quarries I, VI, VII et VIII are opened.

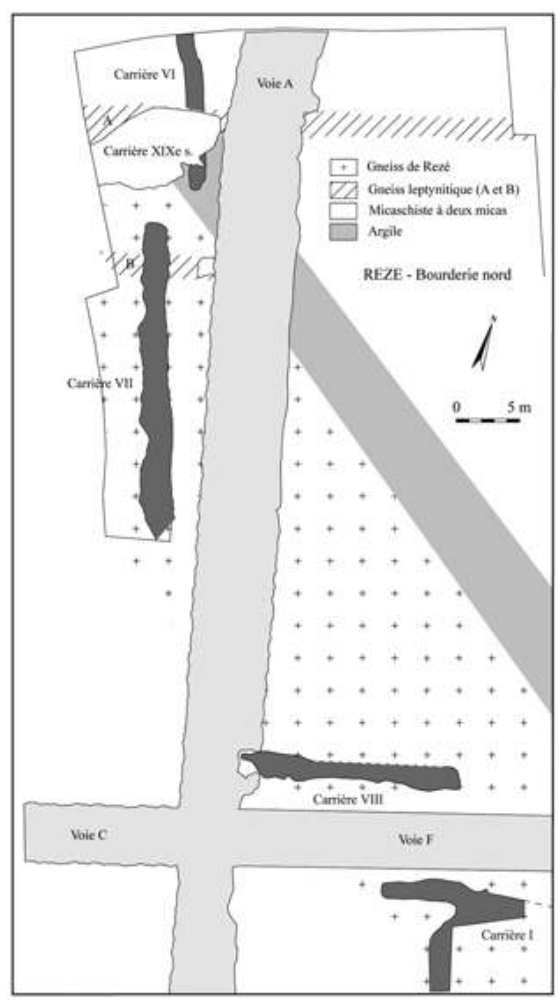

\section{Carrière VII}

La carrière VII, creusée transversalement dans la bande de gneiss précédemment signalée et recoupant une autre bande de gneiss leptynitique cisaillé (B) large de deux mètres et orientée à environ Nord $75^{\circ}$, a été dégagée sur toute sa longueur. Cette excavation d'une longueur de 25 mètres jouxte sensiblement la limite ouest de la voie $\mathrm{A}$ (fig. 7, 8 et 9). D'une largeur de 2 à 3,20 mètres à l'ouverture, sa profondeur varie entre 0,45 mètre au nord, 1,20 mètre au centre et 0,90 mètre au sud. Les différents sondages montrent que les limites du creusement présentent un profil asymétrique en $\mathrm{U}$, avec des parois irrégulières ponctuées d'anfractuosités profondes, conséquences certaines d'un enlèvement de matière guidé par la nature diaclasée du rocher (fig. 10). Certaines diaclases sont affectées de petites cavités ovales de 6 à $7 \mathrm{~cm}$ de largeur et $7 \mathrm{~cm}$ de profondeur, qui pourraient correspondre à des ébauches de logements de coins (fig. 11). On peut estimer de manière approximative que le volume de matériaux extrait se situe entre 22 et 45 mètres cubes. 
Figure 8 : Coupes et implantation des coupes de la carrière VII. DAO L. Pirault. Figure 8: Sections of quarry VII and their location.

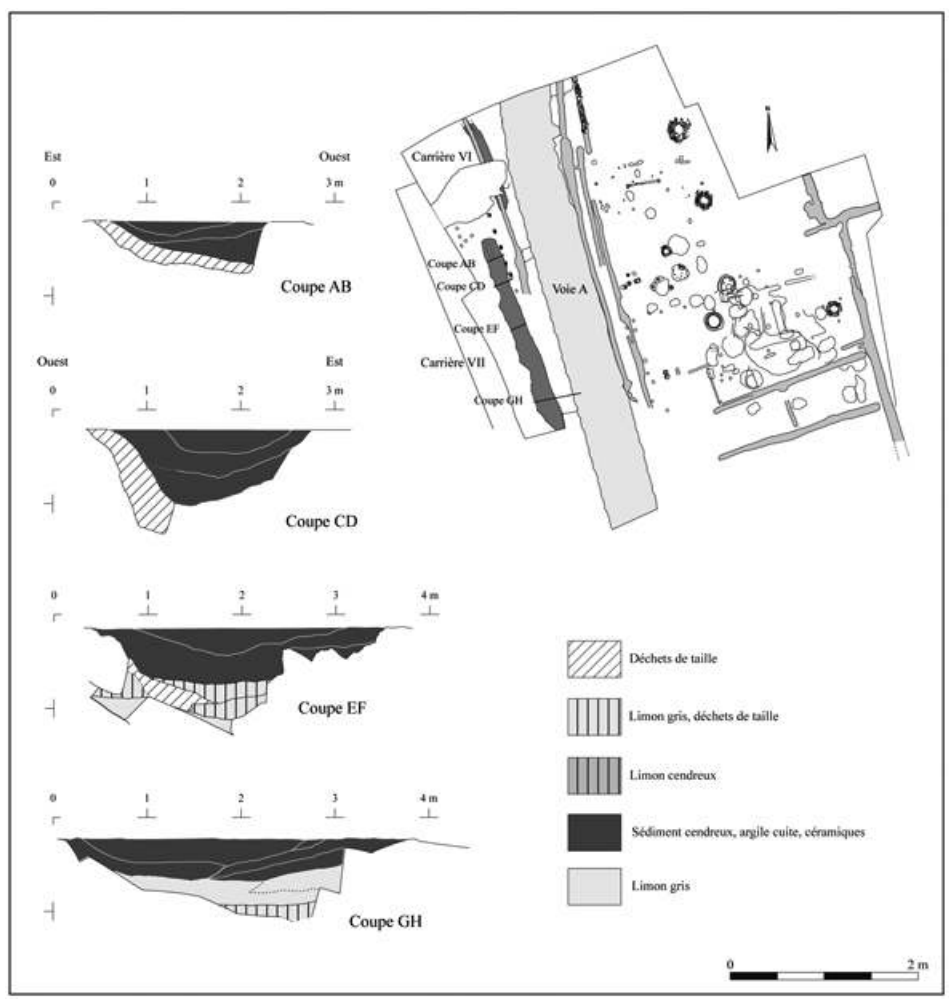

Figure 9 : Carrière VII vue du sud. Cliché L. Pirault. Figure 9 : Quarry VII taken from south.

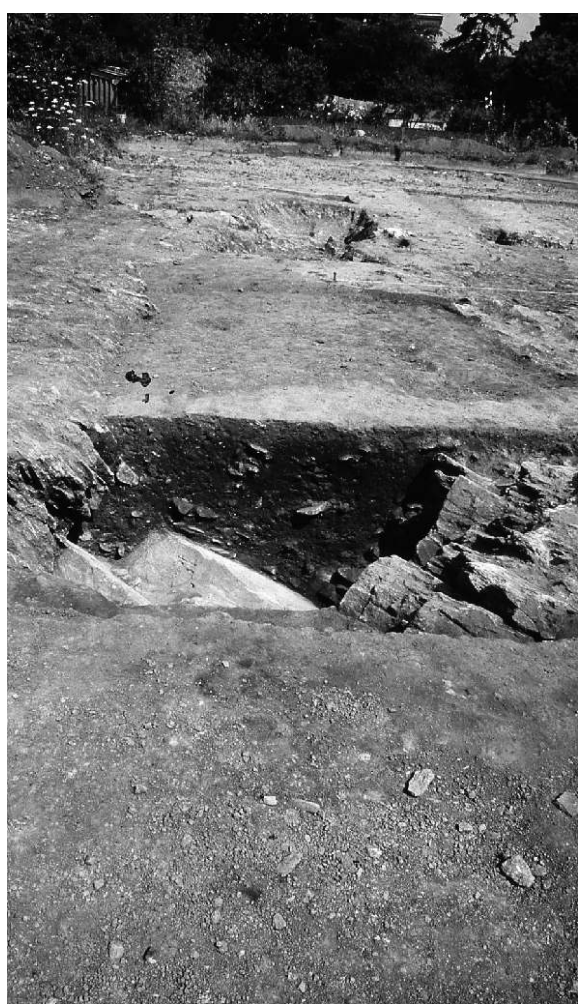


Figure 10 : Paroi de la carrière VII. Cliché L. Pirault. Figure 10: Uneven face of the quarry VII.

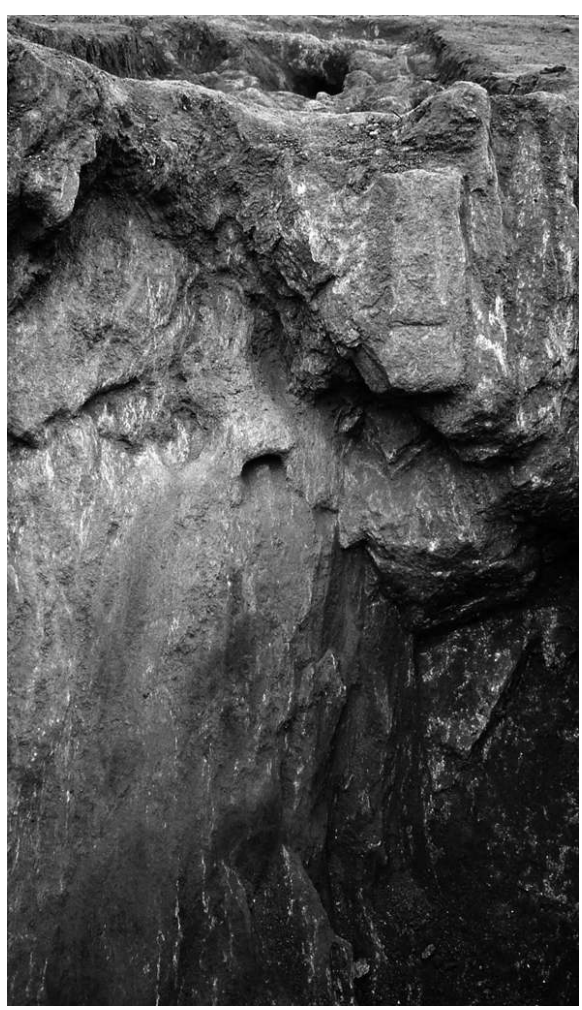

Figure 11 : Probables logements de coins. Cliché L. Pirault. Figure 11: Probable sockets.

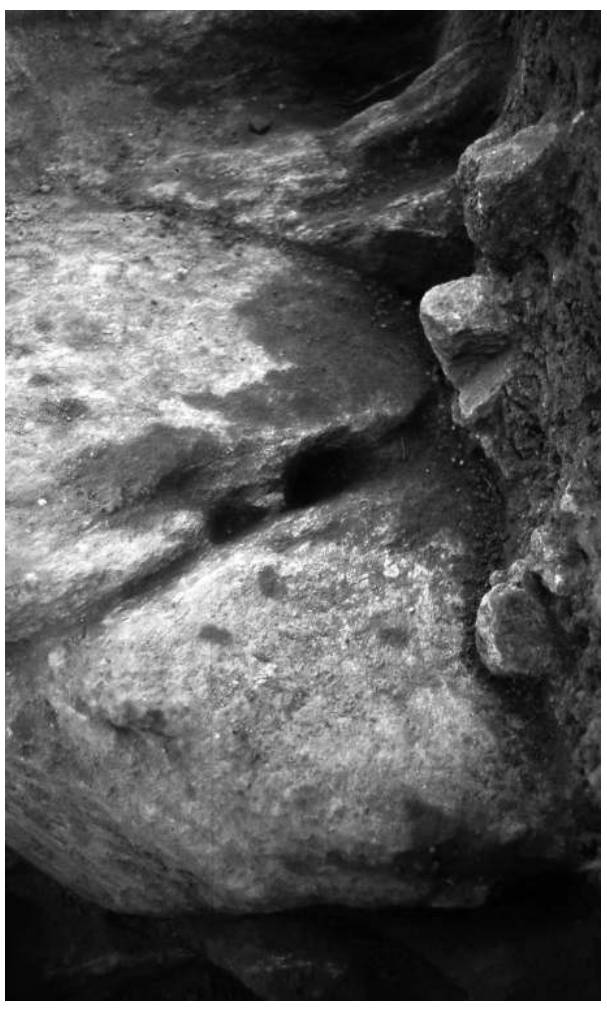


La stratigraphie relevée en plusieurs points montre que la base du comblement est invariablement constituée par des déchets de taille. Ces premiers niveaux sont directement recouverts par des couches de sédiments limoneux et cendreux recélant un abondant mobilier, couvrant la période de 40 à 80 apr. J.-C., avec un fort pourcentage concernant la phase 40-50 apr. J.-C. ${ }^{6}$. La position stratigraphique et la datation du mobilier semblent indiquer que le remblaiement n'excède pas une durée de deux décennies. Si ce dernier débute immédiatement après l'abandon de l'exploitation, vers 40 apr. J.-C., on peut penser raisonnablement que l'activité d'extraction s'est développée dans le premier tiers du $\mathrm{I}^{\mathrm{er}}$ siècle apr. J.-C.

\section{Carrière VIII}

Les voies $\mathrm{C}$ et $\mathrm{E}$ sont reliées par une section de voie $(\mathrm{F})$ très érodée et dont le tracé est perpendiculaire à celui de l'axe de circulation principal (A). Sur la bordure nord de la voie $F$, au niveau de l'intersection entre les voies $A$ et $F$, il a été dégagé une zone d'extraction d'une longueur de 18 mètres (fig. 6, 12 et 13). Les sondages montrent que le fond de cette carrière, d'une largeur moyenne de deux mètres, présente des irrégularités très importantes (de 0,40 à 1,20 mètre) conséquences certaines du débitage du rocher. Certaines diaclases sont affectées de petites cavités ovales de 3 à $5 \mathrm{~cm}$ de profondeur, qui pourraient encore une fois correspondre à des ébauches de logements de coins.

Figure 12 : Coupes et implantation des coupes de la carrière VIII. DAO L. Pirault. Figure 12: Sections of quarry VIII and their location.

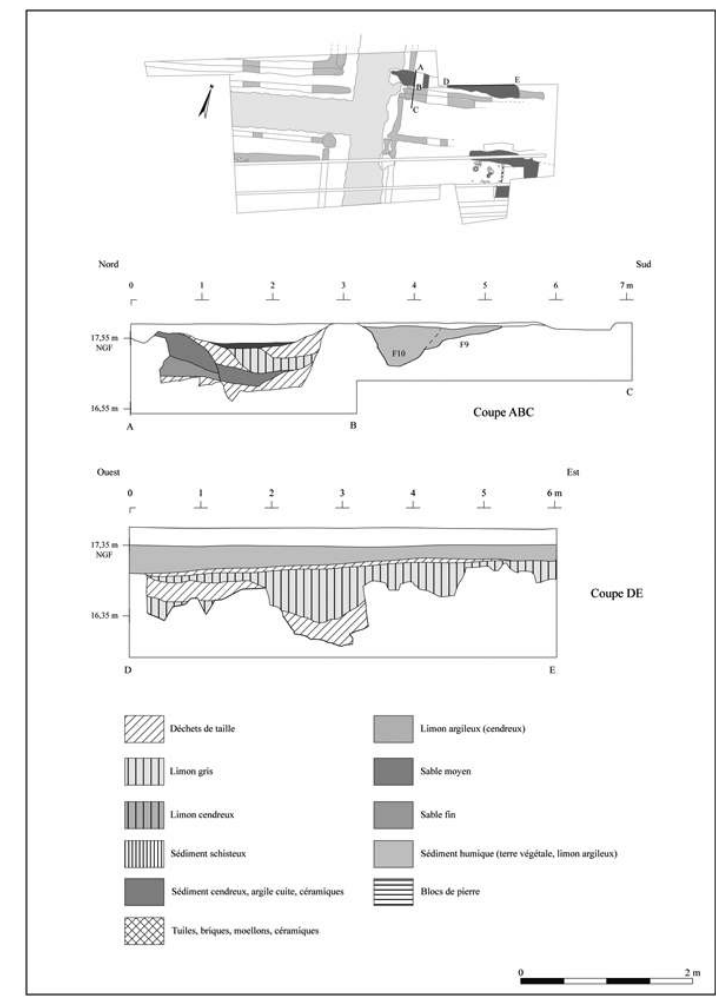


Figure 13 : Carrière VIII vue de l'Ouest. Cliché L. Pirault. Figure 13: Quarry VIII taken from west.

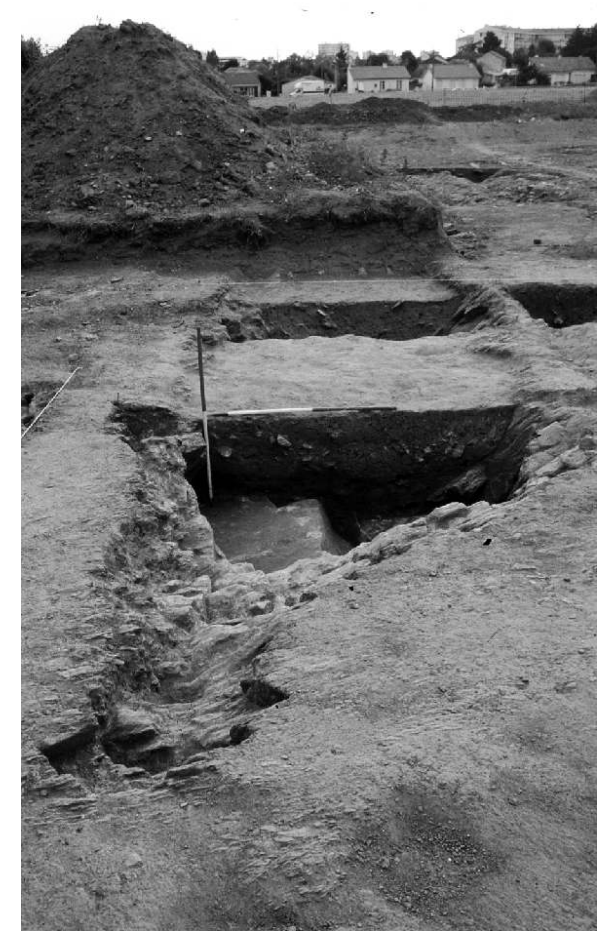

Comme pour la précédente zone d'extraction, la base du creusement est exclusivement occupée par des déchets de taille. Toutefois, des dépôts de ce type de matériaux apparaissent inter-stratifiés entre des strates de sédiments cendreux et limoneux dans la partie la plus occidentale du creusement. Le comblement n'a livré que très peu d'éléments mobiliers. Ces derniers indiquent cependant que le comblement ultime de cette carrière n'est pas postérieur à la fin du i ${ }^{\mathrm{er}}$ siècle apr. J.-C.

\section{Les carrières de Saint-Lupien}

27 Le quartier Saint-Lupien est situé à 300 mètres au nord de la Bourderie. Les campagnes de fouilles programmées entreprises entre 2005 et 2011 sur le site de Saint-Lupien ont permis d'identifier cinq sites d'extraction (Guitton et al., 2005, p. 38-39, fig. ; 2006, p. 27-28, 30-32, fig. ; Arthuis et al., 2008, p. 25-26, 28, fig. ; 2009, p. 49-50, fig. ; Polinski, 2009$, p. 11-13, fig. $)^{7}$. Ces derniers sont tous localisés à l'est de la chapelle Saint-Lupien, dans un espace délimité au nord par un long mur de berge, édifié en bordure de la Loire, et au sud par une rue principale orientée est-ouest (fig. 14). 
Figure 14 : Plan du quartier de Saint-Lupien au $\|^{\mathrm{e}}$ apr. J.-C. avec la localisation des sites d'extraction. DAO M. Monteil.

Figure 14: Plan of Saint-Lupien district in the $2^{\text {nd }}$ century AD with quarries location.

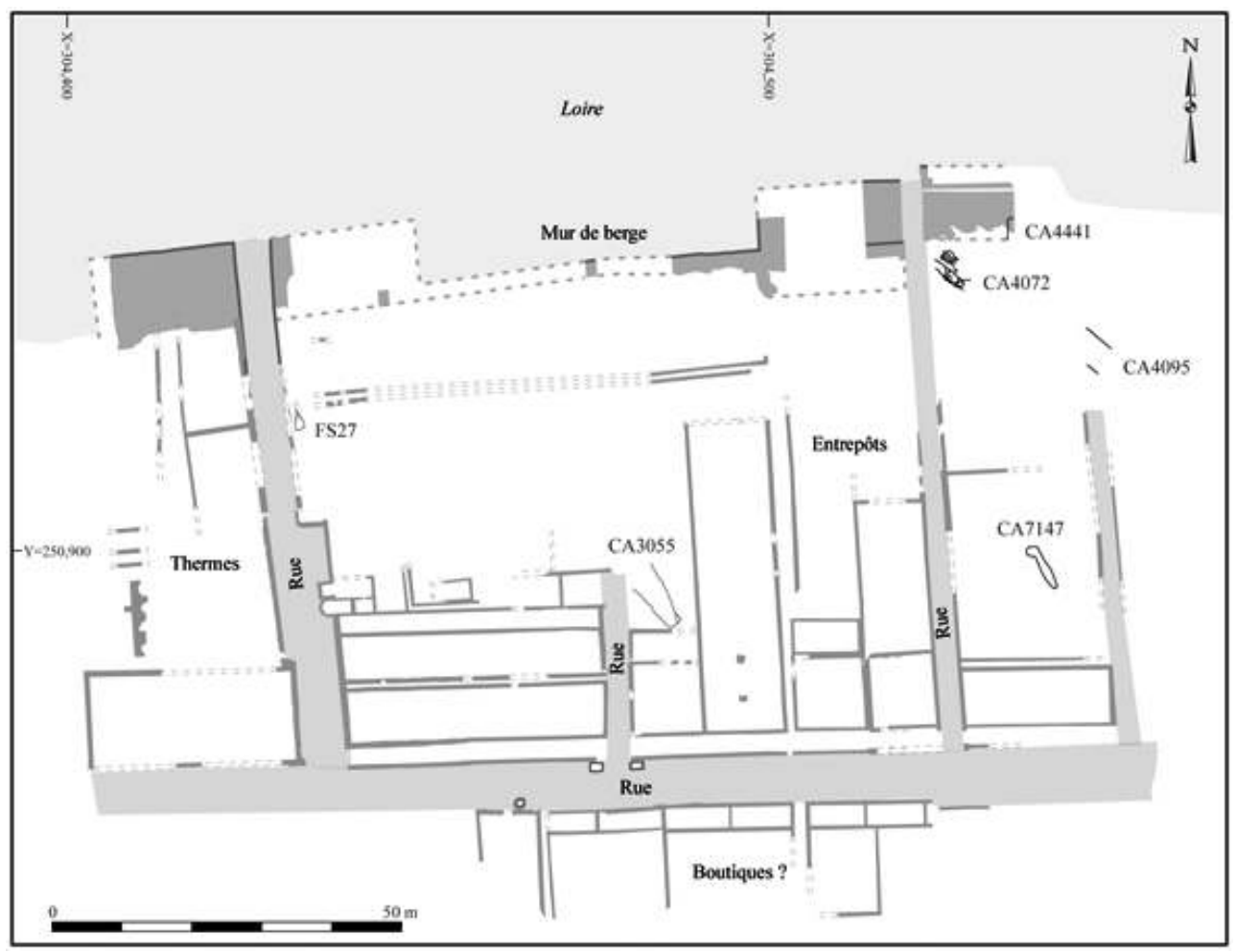

Carrière CA4072

La carrière CA4072 est située à l'est de l'axe de circulation V04374 et à proximité immédiate du mur de berge. Elle est ouverte au niveau d'une bande de granite pegmatitique (US 4165) d'environ 2,30 mètres de largeur, orientée Nord $130^{\circ}$ et bordée de part et d'autre par deux étroites bandes de gneiss de 0,40 mètre (US 4164) et 0,80 mètre (US 4166) de largeur, l'ensemble étant compris entre des micaschistes (US 4163 et 4167) très désagrégés en surface (fig. 15 et 16 ). 
Figure 15 : Implantation des coupes pour les carrières CA4072, CA4095, CA4441, CA3055 et CA7147. DAO A. Polinski, d'après M. Monteil.

Figure 15: Sections location for quarries CA4072, CA4095, CA4441, CA3055 and CA7147.

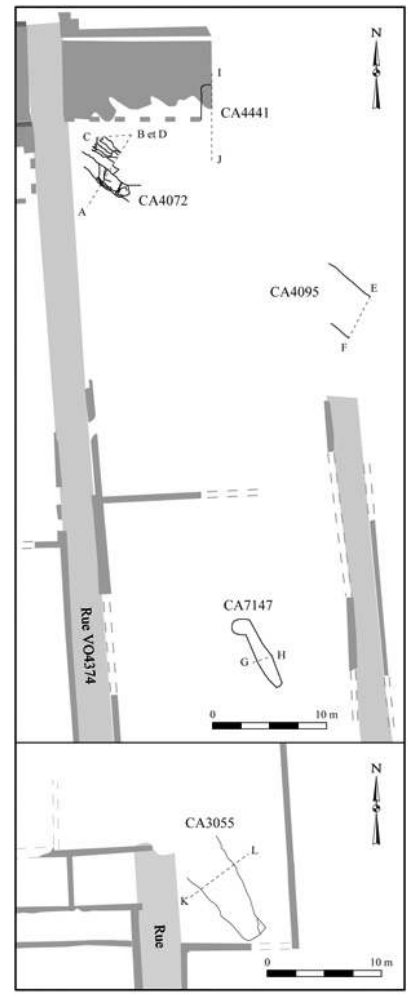

Figure 16 : Coupes des carrières CA4072, CA4095 et CA7147. DAO Équipe de fouille. Figure 16: Sections of quarries CA4072, CA4095 and CA7147.

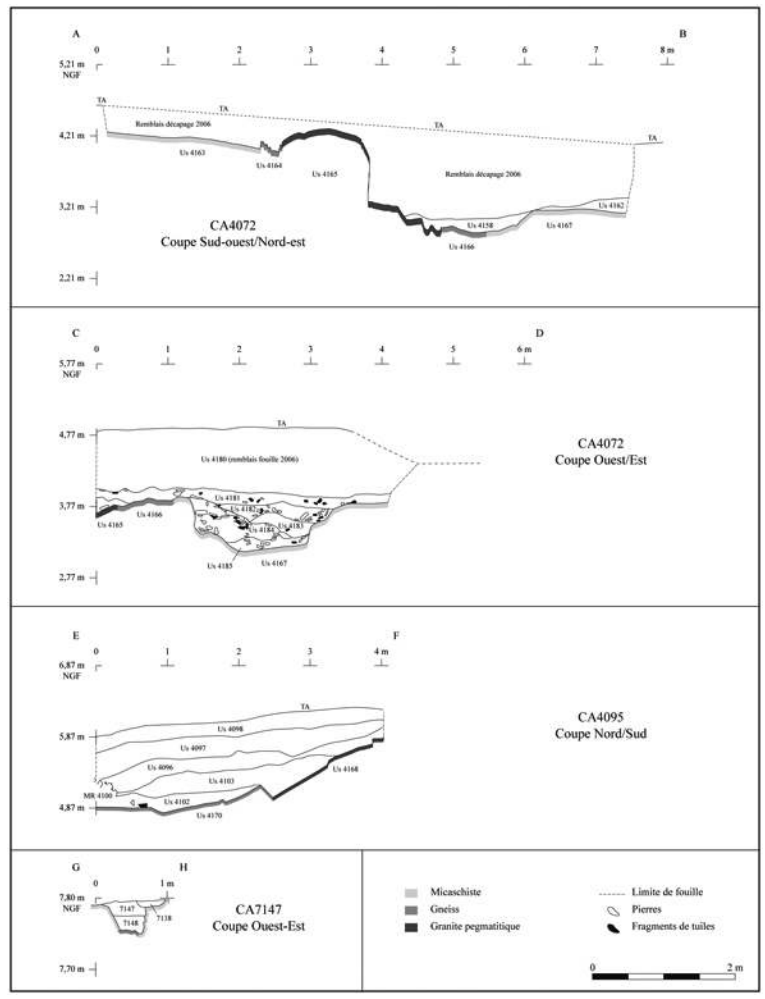


(fig. 17). Elle est en effet marquée, à environ la moitié de sa largeur, par une rupture de pente assez brutale, avec un angle avoisinant $90^{\circ}$, qui contraste fortement avec la pente globale du terrain. Ce phénomène ne trouve pas d'explication géologique satisfaisante aucun indice ne permettant d'envisager l'existence d'une petite faille à ce niveau - et ne peut donc que résulter d'une exploitation méthodique de la bande granitique dans sa partie nord-est et sur plusieurs mètres de longueur. De semblables perturbations du relief apparaissent également le long de son profil nord-ouest/sud-est, mais cette fois sur toute sa largeur, individualisant ainsi un "gros rocher " d'un mètre de hauteur, 1,20 mètre de largeur et un peu plus de 3 mètres de longueur. Ce « rocher» comporte sur son flanc nord-est, sur le dessus et à ses deux extrémités quelques cavités de formes et de dimensions variables qui ne peuvent s'expliquer par un simple phénomène d'érosion, d'altération préférentielle du granite à ce niveau et doivent plutôt être mises en relation avec des prélèvements ciblés de blocs. Le flanc nord-est porte également une succession de six petits paliers de 3 à $10 \mathrm{~cm}$ de hauteur et 5 à quelques dizaines de centimètres de largeur, disposés en escalier et apparaissant en relief sur environ $2 \mathrm{~cm}$ d'épaisseur, certains étant localement dédoublés à hauteur différente (fig. 18). L'inégale largeur et le caractère jointif de ces paliers ne permettent pas d'interpréter ces marques comme les vestiges d'une série d'emboîtures aménagées pour déprendre un large bloc. L'explication la plus plausible reste encore celle d'une figure naturelle obtenue lors du détachement d'un ou plusieurs blocs accolés au « rocher ».

Figure 17 : Carrière CA4072 vue du nord-est. Cliché équipe de fouille. Figure 17: Quarry CA4072 taken from north-east.

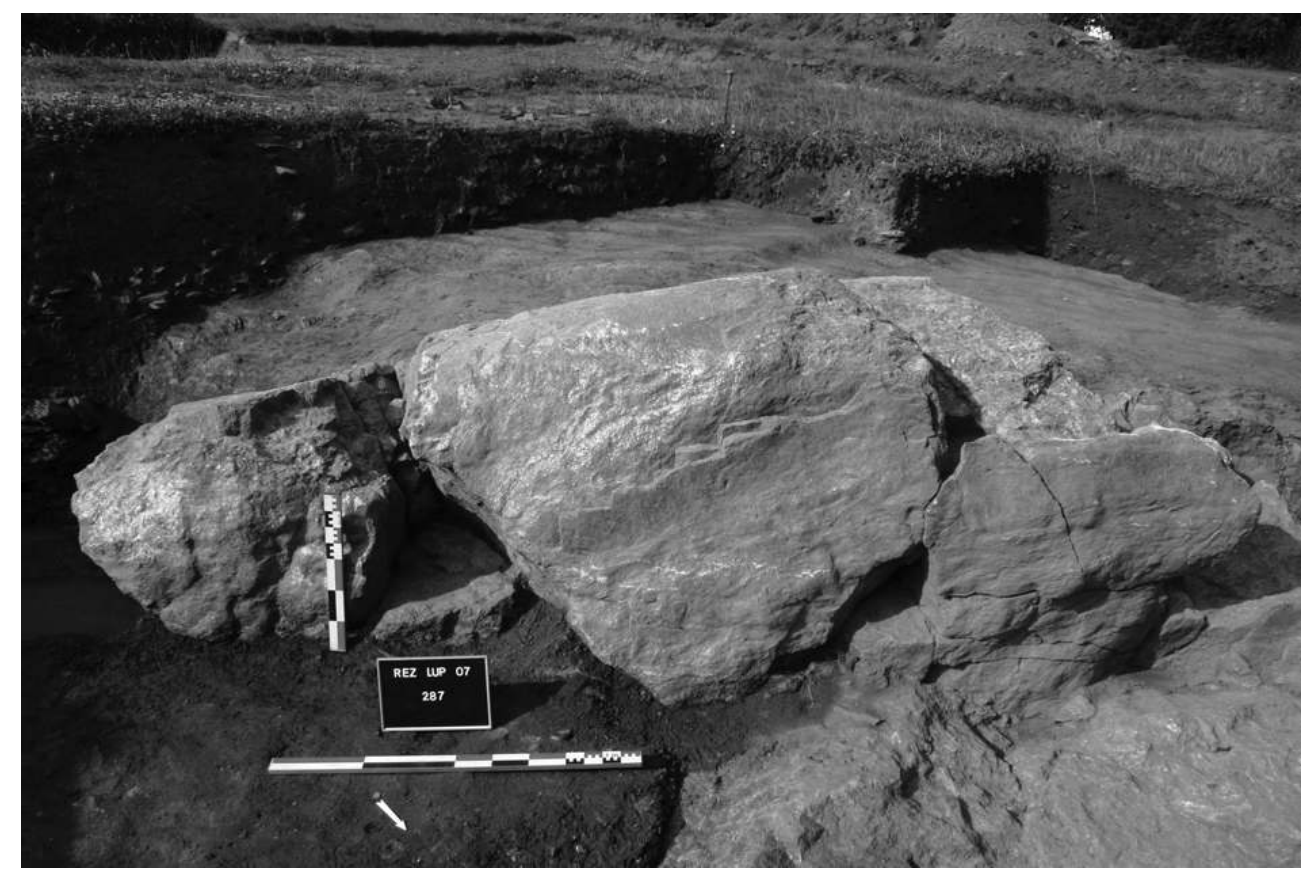


Figure 18 : Carrière CA4072, détail du flanc nord-est du « rocher » de granite pegmatitique. Cliché équipe de fouille.

Figure 18: Quarry CA4072, detail of the north-east side of the "rock" of pegmatitic granite.

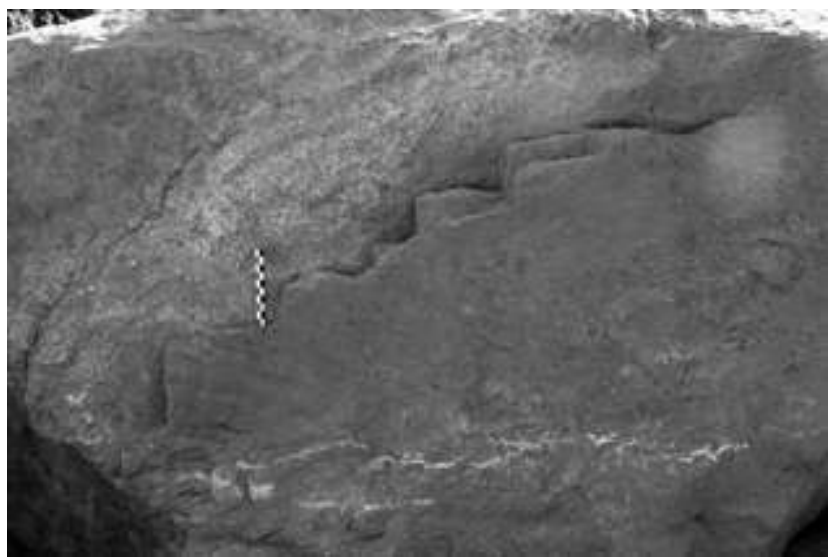

D'autres indices d'extraction se remarquent sur la bordure nord-est de la bande granitique, où la roche est progressivement perturbée par un phénomène de cisaillement (fig. 19). L'exploitation du granite a en effet entraîné des creusements au profil irrégulier, dessinant de longs sillons plus ou moins bien marqués de quelques dizaines de centimètres de largeur. Ce démontage touche aussi la bande de gneiss adjacente (US 4166), en ménageant sur une bonne partie de sa largeur une petite surface plane. Ces différents enlèvements de matière ont été opérés sur au moins 0,30 à 0,60 mètre de profondeur, comme permet d'en juger une petite banquette témoin épargnée par les carriers, quoique entaillée par une série de renfoncements en $\mathrm{V}$. Une petite excavation d'environ deux mètres de largeur et 0,70 mètre de profondeur, dont le fond irrégulier apparaît à environ 3,10 mètres NGF, a en outre été ouverte dans le micaschiste situé immédiatement au nord (US 4167).

Figure 19: Carrière CA4072 vue du sud-est. Cliché équipe de fouille. Figure 19: Quarry CA4072 taken from south-east.

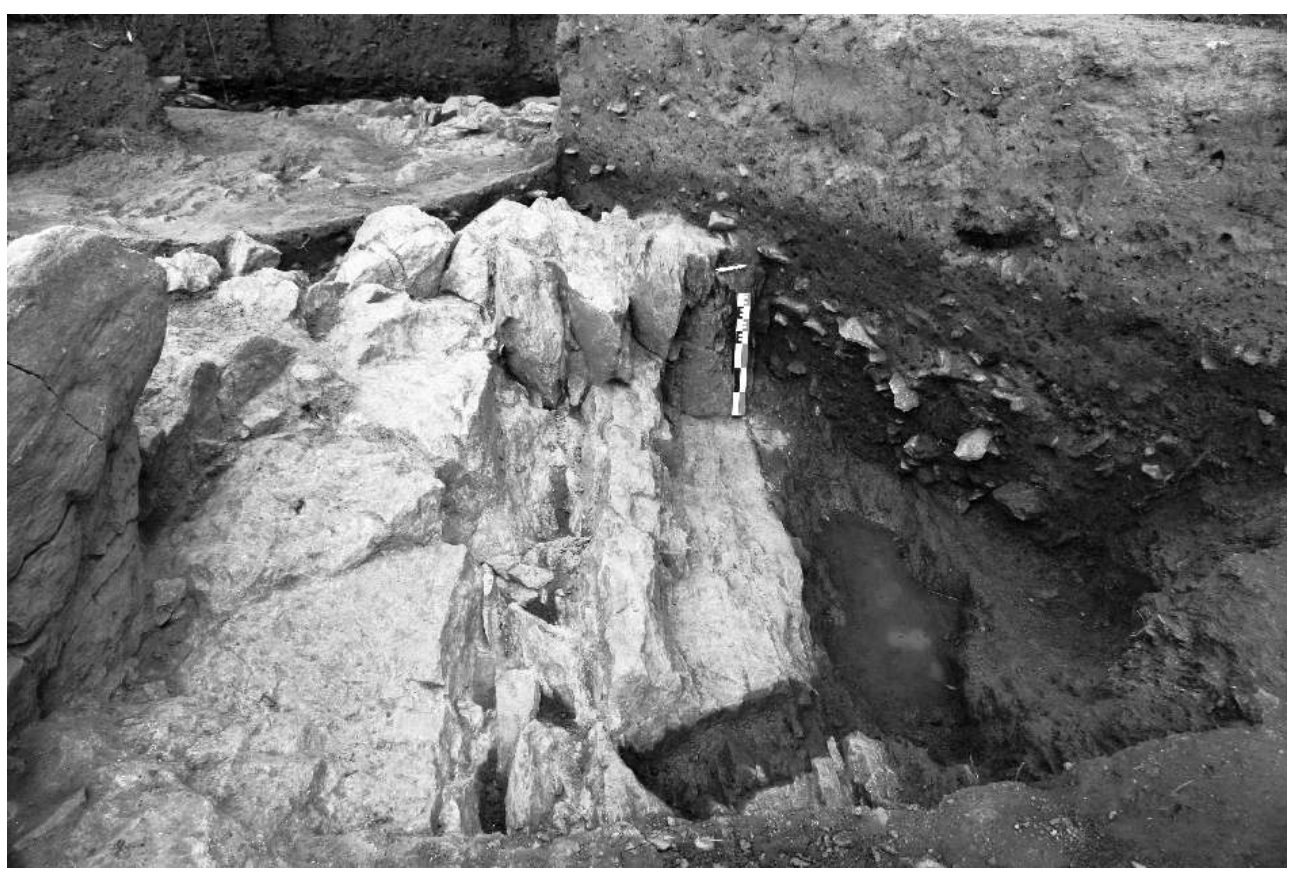


31 Le granite pegmatitique et le gneiss sont fortement diaclasés sur ce site d'extraction. Cette fracturation naturelle confère au gneiss un débit en plaquettes, particulièrement net au niveau de la bande gneissique sud-ouest (US 4164), qui a dû également être mise à contribution dans une moindre mesure, comme le suggère son profil en escalier bien marqué. Les volumes extraits dans la carrière CA4072 sont, d'une manière générale, difficiles à évaluer en raison du manque de données relatives à la physionomie initiale $\mathrm{du}$ substrat, notamment en ce qui concerne la bande granitique. Les niveaux de comblement, limoneux (US 4158, 4162 et 4181 à 4185 ), sont assez riches en plaquettes de micaschiste et de gneiss mais pauvres en mobilier céramique et indiquent un abandon probable de cette zone au cours des années 50-70 de notre ère.

\section{Carrière CA4095}

La carrière CA4095 est ouverte au sud-est de la précédente, à proximité immédiate d'un ancien petit ruisseau aujourd'hui asséché et dans le prolongement des formations précédentes; on retrouve la bande de granite pegmatitique de 2,30 mètres de largeur (US 4168), orientée Nord $130^{\circ}$ et bordée de part et d'autre par deux bandes de gneiss de 2,50 mètres (US 4169) et 2 mètres (US 4170) de largeur (fig. 15, 16 et 20). Elle correspond à un décaissement d'environ 4 mètres de largeur et 0,70 mètre de profondeur, dont le fond irrégulier apparaît à environ 4,87 mètres NGF. L'extraction n'affecte ici que la bande gneissique nord-est (US 4170) et la bande granitique, au niveau de laquelle se remarque localement une petite marche d'escalier d'environ 0,40 mètre de hauteur. Le volume de matériau extrait sur ce site d'extraction, observé seulement sur deux mètres de longueur, peut être estimé à au moins cinq mètres cubes. Les niveaux de comblement, limoneux et incluant quelques plaquettes de micaschiste et de gneiss (US 4096 à 4098, 4102 et 4103), ont livré un mobilier assez pauvre et hétérogène qui permet cependant de situer l'extraction dans le courant du $\mathrm{I}^{\mathrm{er}}$ siècle apr. J.-C. 
Figure 20 : Carrière CA4095 vue du nord. Cliché équipe de fouille. Figure 20: Quarry CA4095 taken from north.

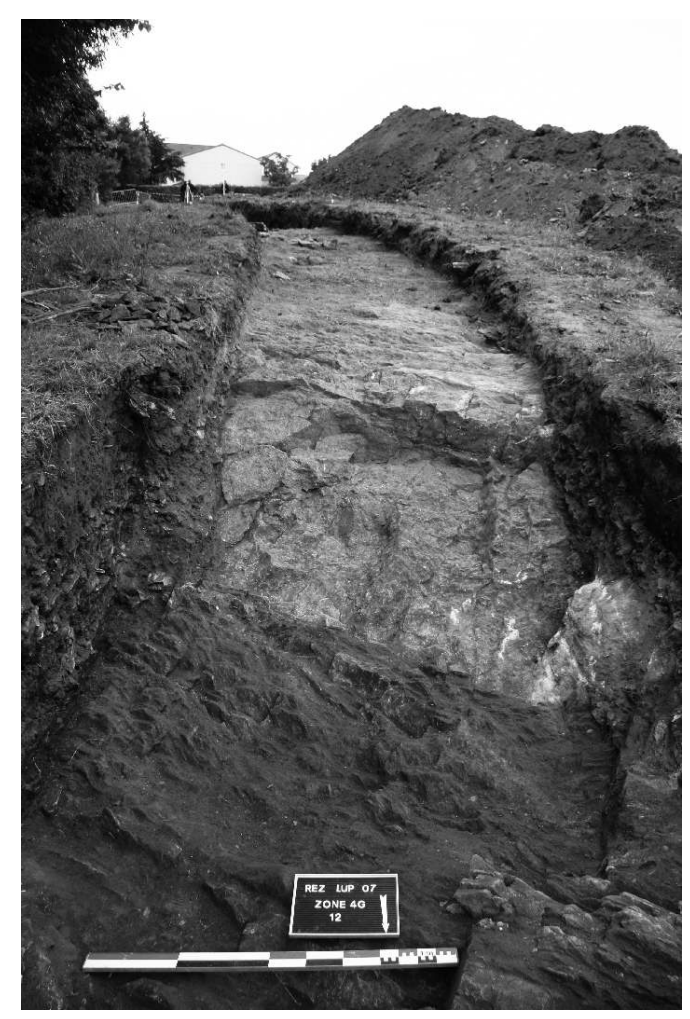

Les carrières CA4072 et CA4095 ne sont vraisemblablement pas des entités indépendantes, mais doivent plutôt représenter les deux extrémités d'un seul et même site d'extraction s'étirant tout le long de la bande de granite pegmatitique, sur une trentaine de mètres environ.

\section{Carrière CA4441}

La carrière CA4441, localisée un peu au nord-est de la carrière CA4072, sous le tracé du mur de berge, n'a guère été étudiée qu'en coupe. Elle se présente sous la forme d'un creusement entaillant le micaschiste sur environ 0,70 mètre de profondeur et au moins 3,75 mètres de largeur et dont le fond irrégulier se situe à environ 1,40 mètre NGF (fig. 15 et 21). Les niveaux de comblement, sableux (US 4284, 4291 à 4293, 4416 à 4418, 4420 à 4423 et 4425), ont livré, outre quelques plaquettes de micaschiste, un peu de mobilier fixant l'abandon dans les années 30-50 de notre ère.

\section{Carrière CA3055}

La carrière CA3055, située à l'ouest du plus grand entrepôt et ouverte presque intégralement dans une large bande de gneiss orientée Nord $140^{\circ}$ (US 3130), n'a été perçue que dans sa partie orientale. Elle se présente comme une tranchée de 2 à 3,60 mètres de largeur, 2,44 mètres de profondeur et au moins une douzaine de mètres de longueur, dont le fond apparaît à 5,24 mètres NGF (fig. 15, 21 et 22). Le volume de matériau extrait dans cette excavation peut être estimé entre 59 et 105 mètres cubes. Les niveaux de comblement, limoneux et incluant localement de nombreux fragments de tuiles et d'enduits peints (US 3127 à 3129) ${ }^{8}$, renferment un abondant mobilier 
céramique, vraisemblablement issu d'un dépotoir d'habitat, qui permet de fixer l'abandon de l'exploitation au cours de la seconde moitié du $\mathrm{I}^{\mathrm{er}}$ siècle apr. J.-C.

Figure 21 : Coupes des carrières CA3055 et CA4441. DAO A. Polinski, équipe de fouille. Figure 21: Section of quarries CA3055 and CA4441.

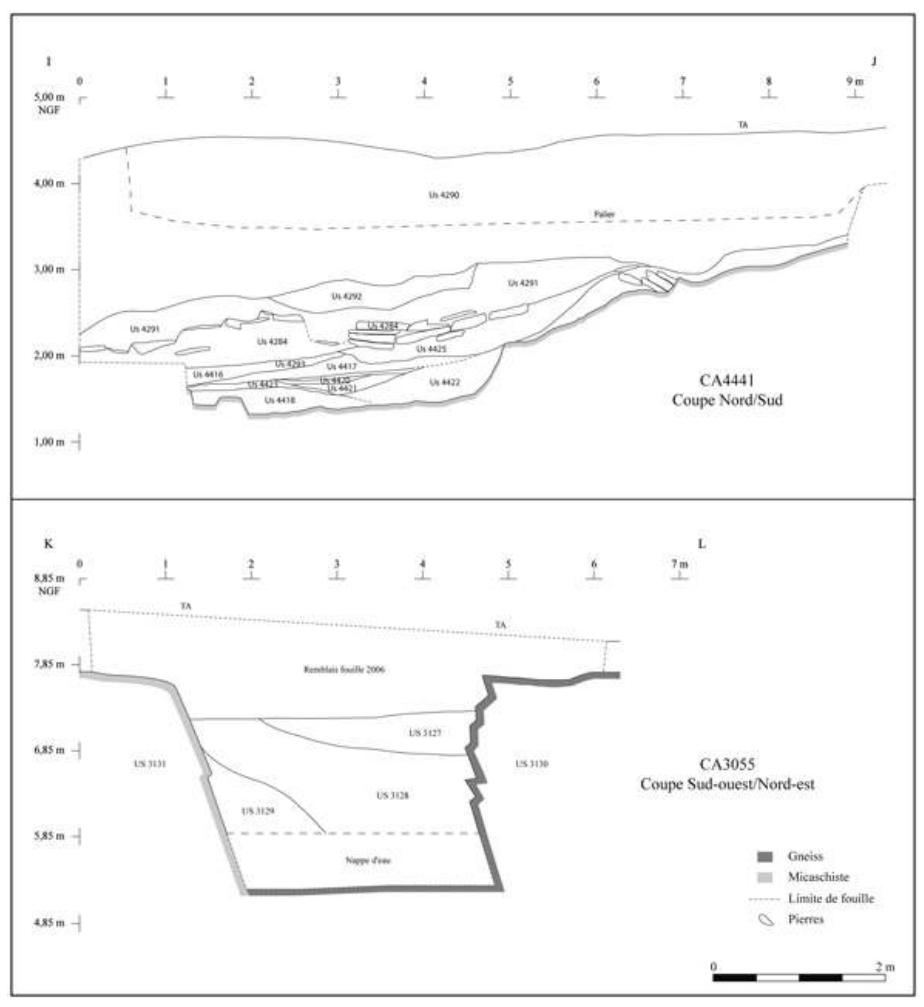

Figure 22 : Carrière $\mathrm{CA} 3055$ vue du sud..

Figure 22: Quarry CA3055 taken from south.

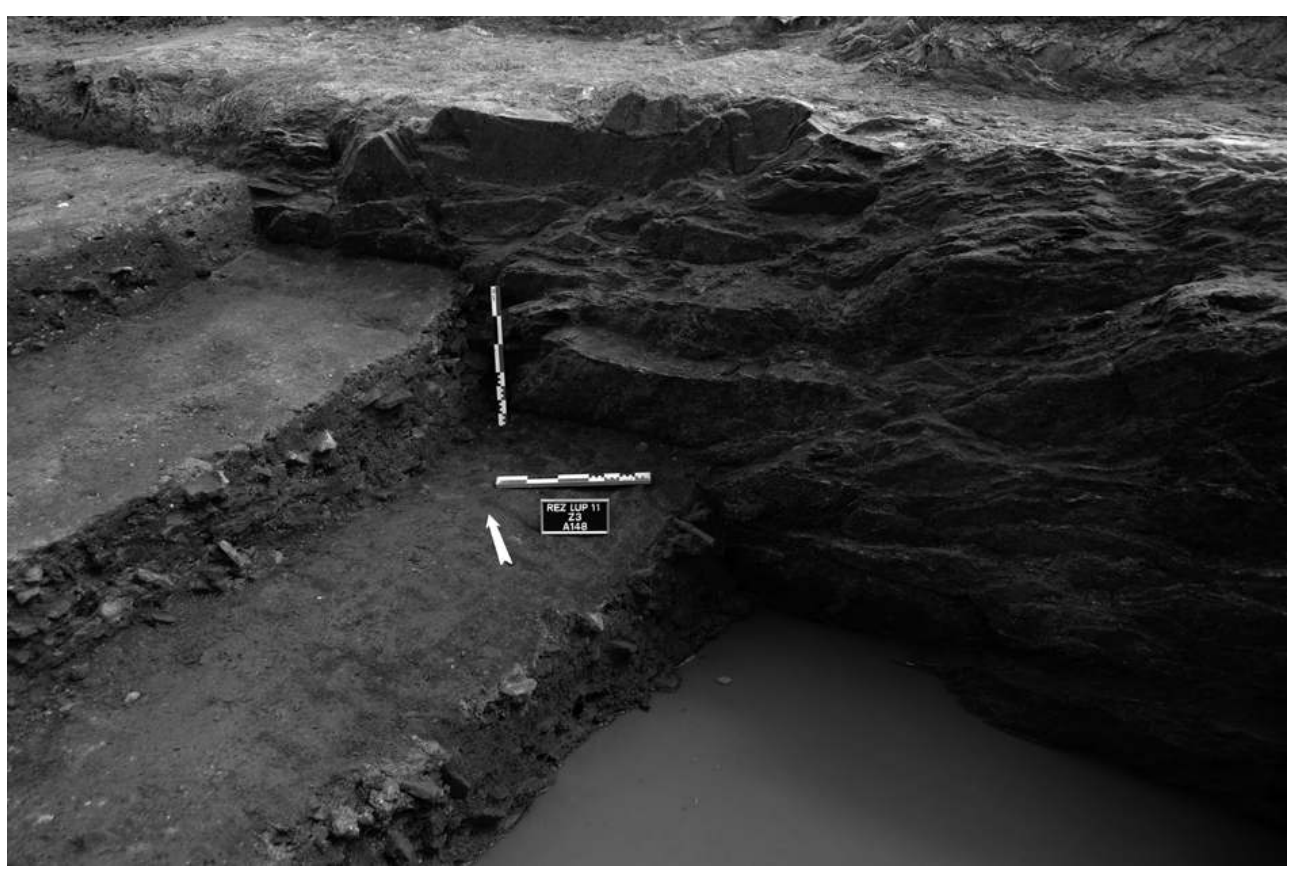

Cliché A. Polinski 


\section{Carrière CA7147}

La carrière CA7147, située à l'emplacement d'un futur entrepôt, constitue un autre exemple de fosse-carrière. Ouverte dans l'axe d'une bande de gneiss orientée Nord $150^{\circ}$, elle s'étend sur 6,70 mètres de longueur et mesure 0,50 à 1,70 mètre de largeur pour 0,40 à 0,90 mètre de profondeur (fig. 15 et 16). Les carriers ont visiblement cherché à extraire spécifiquement ce matériau, puisque le micaschiste qui encadre la bande ne porte pratiquement aucune trace d'exploitation. Le volume extrait sur ce site d'extraction peut être estimé entre 3 et 7 mètres cubes. Les niveaux de comblement, limono-argileux (US 7138, 7147 et 7148), ont livré de nombreux déchets d'extraction et quelques fragments de tuiles, ainsi qu'un peu de mobilier permettant de situer l'extraction dans le courant du $\mathrm{I}^{\text {er }}$ siècle apr. J.-C.

\section{Un sixième site d'extraction sous la chapelle Saint-Lupien ?}

Une autre excavation située à l'intérieur de la chapelle Saint-Lupien, FS27, doit probablement être interprétée comme un site d'extraction de micaschiste (Monteil et al., 2009, p. 9-10) (fig. 14 et 23) ${ }^{9}$. Endommagée par la mise en place d'un pilier de soutènement, cette dépression n'autorise aujourd'hui guère de commentaires et seule sa profondeur permet d'envisager la possibilité qu'elle puisse être rattachée à la période antique. A. Cahour, qui l'a mise au jour en 1872, la décrit cependant comme « une large fosse, de forme ovoïdale et irrégulière et mesurant 2,70 mètres de longueur, du nord au sud » et précise que « sa partie nord, de 0,70 mètre de largeur sur 0,90 mètre de profondeur sous dallage, allait s'agrandissant brusquement vers le sud et donnant à cette dernière partie 1,40 mètre de largeur sur 3,40 mètres de profondeur " (Cahour, 1878, p. 81-82, fig.). Le chanoine, qui s'est interrogé sur la destination de cette fosse énigmatique, rejette l'hypothèse d'un puits et envisage le fait qu'elle ait pu abriter les restes du saint Lupien, tout en reconnaissant lui-même que le profil de ce creusement n'est pas adapté pour une sépulture. L'extraction de pierre destinée à la construction reste donc, en l'état, la seule explication possible à ce décaissement. 
Figure 23 : Fosse FS27 lors des fouilles faites dans la chapelle Saint-Lupien en 1872. D'après Cahour 1878.

Figure 23: Pit FS27 at the time of excavations conducted in Saint-Lupien's chapel in 1872.

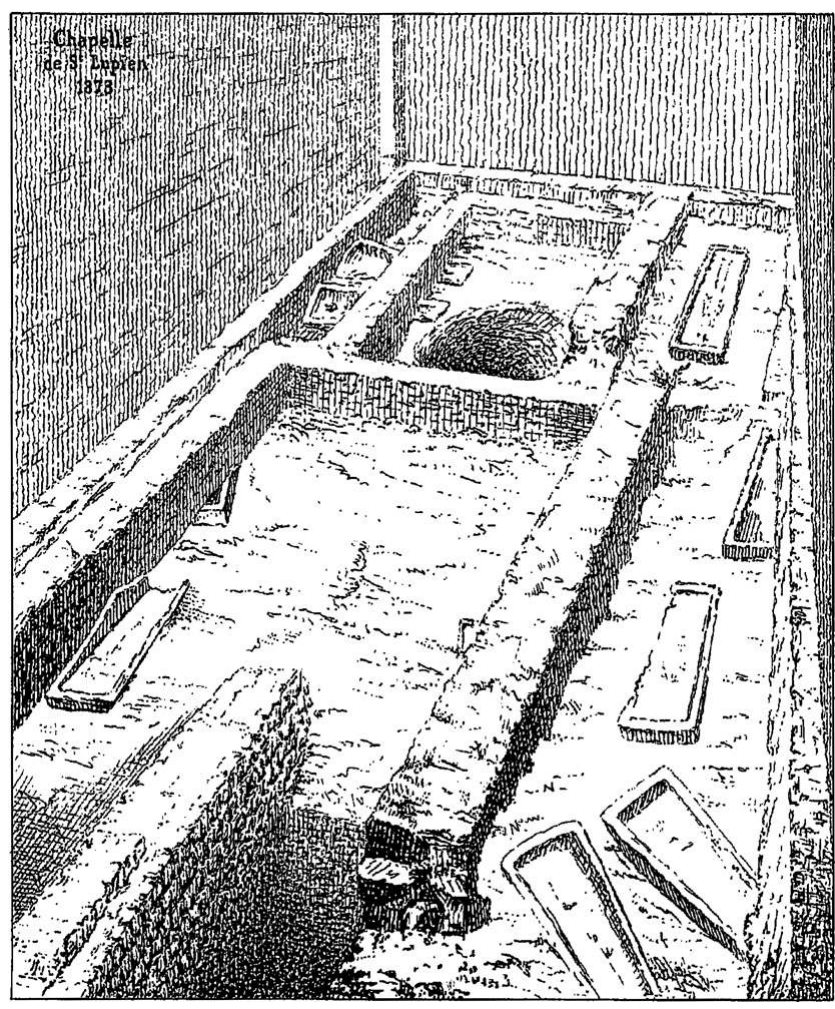

\section{Une possible carrière antique près de la Maison Radieuse}

Une rupture de pente bien marquée associée à une vaste dépression se perçoit dans les terrains compris entre la Maison Radieuse du Corbusier et la voie ferrée reliant Nantes à Pornic, en bordure occidentale d'un lotissement. Cette rupture de pente reste le seul témoin d'un affleurement de près de 150 mètres de longueur, aujourd'hui complètement recouvert de terre et intégré dans des jardins ou espaces verts. Des observations effectuées en 1986, alors que l'affleurement était encore visible, ont permis de remarquer que ce décaissement avait été ouvert dans un granite à grain fin et à deux micas. Cette constatation a son importance, car un leucogranite jaunâtre, à grain fin et à deux micas a été largement mis à contribution dans l'urbanisme de la ville antique à partir de l'époque claudienne, au moment où s'opère le passage d'une architecture en matériaux périssables à une architecture en dur avec des murs maçonnés. Il ne paraît donc pas osé d'envisager la possibilité que cette excavation, qui affecte une formation granitique présente à l'intérieur même de la ville antique, ait pu être exploitée par les Gallo-Romains. Le sol porte en tout cas l'empreinte évidente d'une ancienne carrière, dont l'exploitation doit remonter à une époque fort reculée, puisqu'aucun document d'archives ne semble en avoir conservé le souvenir.

\section{Remarques sur les procédés d'extraction}

Les sites d'extraction fouillés dans les quartiers de la Bourderie et de Saint-Lupien ont livré relativement peu de traces d'outils, hormis quelques probables logements de 
coins, et ce sont surtout les perturbations morphologiques du substrat, la présence de "vides", de petits paliers ou de traces d'arrachements qui témoignent d'une exploitation de la pierre. La nature et les propriétés des roches exploitées excluaient un recours à la technique des havages ${ }^{10}$, mais les carriers pouvaient mettre à profit les nombreuses diaclases affectant le gneiss, le gneiss leptynitique et le granite pegmatitique pour obtenir les moellons, blocs ou dalles recherchés. Il leur suffisait en effet d'introduire dans ces fissures naturelles et de place en place des coins en fer, puis de les forcer à l'aide d'une masse pour entraîner le déchaussement du bloc, en s'aidant au besoin d'une pince de carrier, sorte de barre à mine, pour exercer un effet de levier. L'inégale répartition et les dimensions variables des diaclases ont cependant nécessité le creusement ponctuel d'emboîtures pour permettre la déprise des blocs, comme l'illustrent quelques marques observées à la Bourderie. Ce constat ne concerne cependant que le gneiss, la très grande dureté du granite pegmatitique rendant moins probable le creusement d'emboîtures. L'emploi de coins a dû jouer un rôle prépondérant dans l'exploitation des carrières rezéennes, mais celui du pic d'extraction ne peut être totalement exclu, car son utilisation a pu s'avérer plus judicieuse dans certains cas. Ainsi, le recours aux coins n'apparaît pas toujours nécessaire sur les étroites bandes de gneiss de Saint-Lupien, qui se débitent naturellement en plaquettes de taille variable, et pour lesquels quelques coups de pic bien placés peuvent suffire à déchausser les blocs. Le micaschiste a dû, quant à lui, être extrait selon la technique dite du clivage, qui consiste à introduire des coins dans le plan de schistosité, puis à les forcer pour produire une fente et détacher des dalles d'épaisseur choisie, l'inégale fracturation de la roche pouvant par ailleurs autoriser dans certains cas l'extraction de dalles de grandes dimensions. Le pic n'est sans doute intervenu que pour attaquer les parties les plus superficielles du substrat. Ces modes d'exploitation, basés sur la présence de diaclases ou sur la schistosité de la roche, aboutissent à un résultat forcément aléatoire et expliquent le profil plus ou moins irrégulier des parois et du plancher des sites d'extraction.

Le comblement des carrières de Rezé n'a livré aucun outil pouvant être clairement associé à l'extraction, mais un outil métallique découvert à Saint-Lupien mérite une attention particulière (fig. 24). Cet outil, isolé sous le numéro 6042-1, est malheureusement issu d'un niveau de voirie mal daté, qui contient du mobilier couvrant quasiment toute la période gallo-romaine, et ne peut donc être avec certitude mis en relation avec l'une des carrières de ce quartier. D'une hauteur totale de $13,2 \mathrm{~cm}$ pour un poids de $200 \mathrm{~g}$, il se compose d'une tête de $3,26 \mathrm{~cm}$ de largeur, d'une partie cylindrique de $2 \mathrm{~cm}$ de diamètre et environ $5 \mathrm{~cm}$ de hauteur et d'un tranchant de $8 \mathrm{~cm}$ de hauteur, allant en s'amincissant rapidement et s'élargissant progressivement pour atteindre $6 \mathrm{~cm}$ de largeur et $2 \mathrm{~mm}$ d'épaisseur. Cet instrument se rattache clairement à la catégorie des outils à percussion posée avec percuteur, ce que confirment l'aplatissement et la déformation assez marqués de la tête. On a récemment proposé de l'interpréter comme un ciseau à tranchant droit (Bertrand, 2009, p. 6, fig.), sur la base d'objets assez proches conservés au musée d'Avenches (Suisse) et donnés comme ayant pu servir au travail de la pierre (Duvauchelle, 2005, p. 63-64, 177-180, fig.). La morphologie de l'objet ne parait cependant pas compatible avec une telle interprétation et plaide plutôt en faveur d'un coin; il s'agirait donc d'un outil de carrier et non d'un outil de tailleur de pierre ${ }^{11}$. Il est en effet difficile d'affirmer que l'extrémité du tranchant était droite à l'origine, comme sur la majorité des ciseaux (Bessac, 1993, p. 121-137, fig.), car rien ne prouve que la forme légèrement arrondie soit 
le résultat d'une forte usure. Le corps de l'outil est par ailleurs très court et n'est pas adapté à une préhension à pleine main, comme le font traditionnellement les tailleurs de pierre pour bien guider le ciseau. Cette faible longueur pourrait s'expliquer par les coups violents et répétés portés sur la tête de l'outil, mais cela signifierait que ce dernier ait continué à être utilisé malgré un stade d'usure avancé ne permettant déjà plus une bonne prise en main. Un ciseau aurait enfin comporté au niveau de son tranchant des côtés rectilignes, au moins sur une certaine partie de sa hauteur, ce qui n'est pas le cas ici. Cet outil, qu'il semble donc possible d'assimiler à un coin, n'a pu en aucun cas servir pour débiter directement des blocs de granite ou de gneiss par insertion dans des emboîtures préalablement creusées, car cette opération aurait nécessité des coins moins larges, plus massifs et avec des faces biseautées (Bessac et al., 1996, p. 214-217, fig.). Il a pu en revanche être introduit dans les diaclases affectant ces roches ou être utilisé pour l'extraction du micaschiste. Le procédé consisterait alors à cliver le micaschiste à l'affleurement selon son plan de schistosité, afin d'individualiser des dalles d'épaisseur choisie. La largeur et la hauteur relativement importantes de la surface active et son profil effilé sont en effet adaptés pour un tel emploi. L'outil n'a pu en revanche, pour les raisons déjà évoquées, servir à fendre des dalles déjà extraites et être utilisé comme un ciseau de querneur (Linden, 2004, p. 110-111, 204, 206, fig.) ${ }^{12}$. L'hypothèse d'une utilisation pour le travail du bois ou d'une autre matière ne peut certes être écartée, mais la présence dans le voisinage de plusieurs sites d'extraction permet tout de même d'envisager sérieusement un lien entre cet instrument et le travail de la pierre.

Figure 24 : Probable coin en fer découvert à Saint-Lupien. DAO A. Polinski, d'après A. Archer. Figure 24: Probable iron wedge from Saint-Lupien.

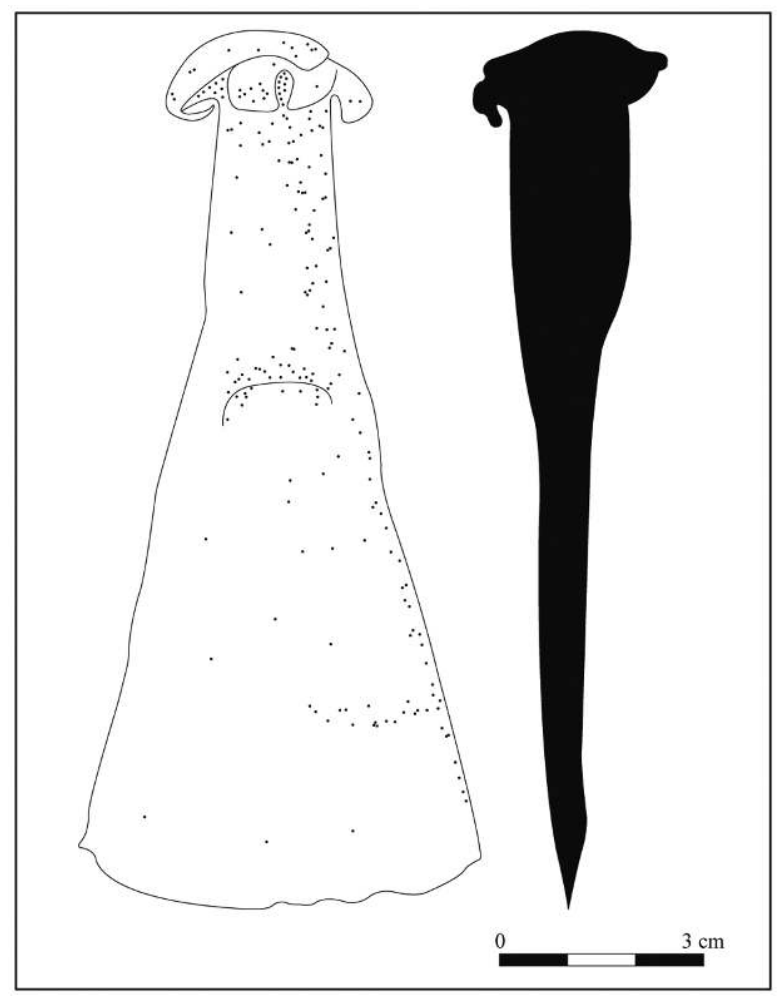




\section{Le devenir des produits d'extraction}

41 Si un simple examen macroscopique suffit pour constater la similitude entre les roches constituant le substrat, c'est-à-dire le gneiss, le micaschiste, mais aussi le granite pegmatitique et le gneiss leptynitique, et celles mises en œuvre dans la voirie ou les divers aménagements construits de Saint-Lupien et de la Bourderie, les quelques carrières mises au jour fournissent une preuve irréfutable de l'exploitation des ressources locales. Cette exploitation est d'ailleurs plutôt déterminée par la nature géologique du substrat que par la volonté d'exploiter ces roches en particulier. La présence anecdotique de moellons ou de blocs d'amphibolite dans plusieurs maçonneries de Saint-Lupien doit de même s'expliquer par le fait que des bandes d'amphibolite - peut-être celles évoquées plus haut, même si l'absence de fouille extensive ne permet pas encore de le vérifier - ont été pour une raison donnée, mais sans doute par hasard, touchées par l'extraction. D'autres roches sont bien sûr employées dans ce quartier, mais il serait hors de propos de discuter de leur origine et des modalités de leur utilisation, ces matériaux ne provenant pas des carrières précédemment décrites.

L'ensemble de ces sites d'extraction a, d'une manière globale, fonctionné au cours du $\mathrm{I}^{\mathrm{er}}$ siècle apr. J.-C. à l'exception peut-être des carrières II et III de la Bourderie, dont le comblement ne semble pas intervenir avant la fin et le milieu du II $^{\mathrm{e}}$ siècle. Ils n'ont pour autant pas tous été ouverts en même temps, ni alimenté les mêmes chantiers. À SaintLupien, les carrières CA3055, CA4072, CA4095 et CA7147 ont dû fournir une partie de la pierre nécessaire aux constructions ou aux divers aménagements antérieurs au $\mathrm{II}^{\mathrm{e}}$ siècle de notre ère, en particulier pour les solins, radiers, égouts et la voirie. La carrière CA4072 peut être également en lien avec le long mur de berge construit dans les années 50-70 apr. J.-C. quasi exclusivement avec d'épaisses dalles de micaschiste, mais elle n'a pu cependant à elle seule procurer l'énorme volume de pierre qu'a exigé cet aménagement monumental de plus de 150 mètres de longueur. Le mur de berge a de toute évidence nécessité l'ouverture d'une plus vaste carrière et les quelques sites d'extraction recensés à ce jour ne donnent qu'une vision extrêmement partielle de l'exploitation de la pierre à Saint-Lupien. Le comblement de CA4441, plus précoce, exclut quant à lui tout lien entre cette carrière de micaschiste et le mur de berge et incite plutôt à la mettre en relation avec les premiers aménagements de l'époque tibérienne. Dans tous les cas, il n'est pas possible, en l'absence de données chronologiques suffisamment précises, d'établir un lien direct avec un ou des aménagements particuliers. À la Bourderie, les matériaux extraits des carrières VI, VII et VIII semblent avoir été mis à contribution pour l'aménagement et la remise en état des voies $\mathrm{A}$ et $\mathrm{F}$, construites respectivement vers 25-30 apr. J.-C. et dans la première moitié du II ${ }^{\mathrm{e}}$ siècle. La fouille d'un tronçon de la voie A sur une longueur de 75 mètres a en effet permis de constater que le radier de l'aire de circulation, large de 7 mètres, est presque intégralement formé de blocs de gneiss d'origine locale. Le lien entre les carrières de ce quartier et la voirie peut peut-être expliquer la présence du gneiss leptynitique à Saint-Lupien, où celui-ci se concentre essentiellement au niveau des axes de circulation.

Les produits des différentes carrières n'ont, à l'exception du micaschiste, pas fait l'objet de traitement particulier après extraction. Les blocs de gneiss et de granite pegmatitique de trop grandes dimensions ont pu éventuellement être débités en blocs 
plus petits, mais l'importante fissuration de ces roches ne devait pas toujours rendre nécessaire ce débitage. Les moellons ou blocs obtenus ont été mis en œuvre sans taille préalable - la nature de la roche ne s'y prêtant pas vraiment ou pas du tout - ou tout au plus après un léger équarrissage pour supprimer quelques excroissances. Lorsque le mur comportait une face parementée, il suffisait aux maçons d'opérer un rapide tri, comme cela se fait encore aujourd'hui, pour isoler des moellons ou blocs dotés d'une face naturellement plus plane ou plus régulière, lesquels étaient ensuite utilisés pour le parement. Les dalles de micaschiste ont en revanche subi une rapide mise en forme, comme le montrent les observations menées au niveau du mur de berge (Guyodo, 2010, p. 1-6, fig.). Un certain nombre d'entre elles portent en effet, sur un ou plusieurs côtés, un nombre variable de traces d'impacts en $\mathrm{V}$, qui résultent d'une volonté d'éliminer les parties jugées trop saillantes et de régulariser certaines arêtes. Les coups sont portés de biais avec la partie plate du marteau têtu, la partie pointue de l'outil pouvant servir dans un second temps à parfaire la face de parement. Certaines dalles de dimensions trop importantes ont pu éventuellement être coupées en deux grâce à une technique assez simple, qui consiste à tracer sur une face une ligne peu profonde, perpendiculairement au plan de schistosité, puis à frapper sur l'autre face à l'aide d'un maillet pour provoquer la rupture et obtenir un plan de cassure assez régulier.

L'ensemble des sites d'extraction reconnus à la Bourderie et à Saint-Lupien n'a clairement qu'un intérêt local. Il est d'ailleurs peu probable que la diffusion du produit de ces carrières ait dépassé l'échelle du quartier, d'autres petits sites d'extraction similaires répartis sur l'ensemble de l'agglomération ayant dû répondre aux besoins des différents quartiers. Aucun indice ne renseigne sur les hommes qui y ont travaillé, mais la nature des roches exploitées et les procédés d'extraction qu'elles ont conditionné ne nécessitaient pas de bagage technique particulièrement élevé dans le domaine de l'extraction et il est vraisemblable que les mêmes individus exploitaient les carrières et intervenaient sur les chantiers de construction voisins. On aurait donc plutôt affaire à des maçons s'improvisant carriers au gré des besoins en pierre.

La forme allongée et la situation topographique de certaines carrières de la Bourderie ne sont assurément pas le fait du hasard, mais s'expliquent certainement par le lien que celles-ci entretiennent avec la voirie. On ne peut totalement exclure qu'il s'agisse de zones d'extraction relevant de propriétaires riverains chargés de l'entretien des voies. Mais, cette configuration pourrait aussi être liée à l'existence d'une stricte obligation de servitude, qui résulterait d'un ensemble de règles applicables à la collectivité publique. Celles-ci confineraient le long des voies, c'est-à-dire dans le domaine public, l'extraction des matériaux destinés à la construction et à l'entretien de la voirie. Un exemple assez similaire existe à Cussy-les-Forges, dans l'Yonne, où une carrière de granite se présentant sous la forme d'une tranchée de 400 mètres de longueur, 12 mètres de largueur et 3 mètres de profondeur a été ouverte pour l'aménagement d'un tronçon de la voie d'Agrippa, qui relie Avallon à Saint-Moré (Bedon, 1984, p. 39). Il est délicat d'affirmer que les carrières de Saint-Lupien appartiennent éventuellement, elles aussi, au domaine public, mais cette possibilité doit être envisagée, au moins pour certaines d'entre elles, car l'édification du mur de berge ne peut résulter que d'un programme édilitaire. 


\section{Conclusion}

L'agglomération secondaire de Rezé a donc livré une douzaine de sites d'extraction de taille modeste, ouverts principalement dans des micaschistes et des gneiss et ayant fonctionné pour la plupart dans le courant $d u \mathrm{I}^{\mathrm{er}}$ siècle apr. J.-C. Les procédés d'extraction employés, adaptés à la schistosité ou à l'intense fracturation de la roche, devaient laisser une large place à l'utilisation du coin, dont un exemplaire a peut-être été retrouvé sur le site de Saint-Lupien. Ces petites carrières n'ont qu'un intérêt strictement local, puisqu'elles n'ont servi qu'à fournir la pierre nécessaire aux bâtiments et autres aménagements des quartiers de la Bourderie et de Saint-Lupien, mais leur étude soulève néanmoins un certain nombre de questionnements d'ordre technique, économique, social et législatif. Il convient à ce titre de ne pas négliger ce type de sites d'extraction.

\section{BIBLIOGRAPHIE}

ADAM J.-P., 2011 - La construction romaine, Matériaux et techniques, 6e édition, Paris, A. et J. Picard (coll. « Grands manuels Picard »), 368 p., fig.

ARTHUIS R. et al., 2007 - « Nouvelles recherches sur le port fluvial et les entrepôts antiques de Rezé (Loire-Atlantique) », Aestuaria, 12, p. 129-138, fig.

ARTHUIS R. et al., 2008 - L'agglomération antique de Rezé (Loire-Atlantique), le quartier Saint-Lupien, fouille programmée 2006-2008, Rapport intermédiaire 2007, 2 vol., 46 p., fig. (Archives scientifiques du Service régional de l'archéologie des Pays de la Loire)

ARTHUIS R. et al., 2009 - L'agglomération antique de Rezé (Loire-Atlantique), le quartier Saint-Lupien, fouille programmée 2006-2008, rapport final 2008, tome I, 2 vol., 78 p., fig. (Archives scientifiques du SRA des Pays de la Loire).

ARTHUIS R. et al., 2010a - « Archéologie portuaire estuarienne entre Loire et Seine : principaux résultats et questions d'ordre méthodologique, L'exemple des sites antiques d'Aizier (Eure) et de Rezé (Loire-Atlantique) », in HUGOT L. et TRANOY L. (dir.), Les structures portuaires de l'Arc atlantique dans l'Antiquité : bilan et perspectives de recherche, Aquitania, Suppl. 18, p. 61-82, fig.

ARTHUIS R. et al., 2010b - « D’un estuaire à l'autre, Géoarchéologie comparée des ports antiques d'Aizier et de Rezé ", Archéopages, 30, p. 26-35, fig.

BEDON R., 1981 - Les carrières et les carriers de la Gaule romaine, Thèse de doctorat en études latines, université de Tours, sous la direction de R. Chevalier, 319 p., fig.

BEDON R., 1984 - Les carrières et les carriers de la Gaule romaine, Paris, Picard, 247 p., fig. BERTRAND I., 2009 - « Mobilier en métal (alliage à base de cuivre, fer, plomb), os et verre, fouilles 2006-2008, étude préliminaire », in ARTHUIS R. et al., L'agglomération antique de Rezé (LoireAtlantique), le quartier Saint-Lupien, fouille programmée 2006-2008, rapport final 2008, tome II, p. 2-10, fig. (Archives scientifiques du SRA des Pays de la Loire). 
BESSAC J.-C., 1993 - « L'outillage traditionnel du tailleur de pierre de l'Antiquité à nos jours », Revue archéologique de Narbonnaise, Suppl. 14, 319 p., fig.

BESSAC J.-C., 2004 - « Pierre de taille : archéologie et technique », in FERDIÈRE A. (dir.), La construction, les matériaux durs : pierre et terre cuite, Paris, Errance (coll. « Archéologiques »), p. 7-49, fig.

BESSAC J.-C. et al., 1996 - « La pierre en Gaule narbonnaise et les carrières du Bois des Lens (Nîmes), Histoire, archéologie, ethnographie et techniques ", Journal of Roman Archaeology, Suppl. 16, 319 p., fig.

BESSAC J.-C. et SABLAYROLLES R. (dir.), 2002 - « Carrières antiques de la Gaule, Une recherche polymorphe », Gallia, 59, p. 1-204, fig.

CAHOUR A., 1878 - « Recherches archéologiques et hagiographiques sur Saint-Lupien de Rezé », Bulletin de la Société archéologique de Nantes et du département de la Loire-Inférieure, 17, p. 75-97, fig. DESCHAMPS s. et al., 1992 - « Ratiatum (Rezé, Loire-Atlantique) : origines et développement de l'organisation urbaine », Revue archéologique de l'Ouest, 9, p. 113-127, fig.

DeSChampS S. et PIRAUlT L., 1999 - « Rezé, cité des Pictons », Archéologia, 353, p. 34-45, fig.

DUVAUCHELlE A., 2005 - Les outils en fer du Musée romain d'Avenches, Avenches, Pro Aventico (coll. « Documents du Musée Romain d'Avenches », 11), 232 p., fig.

EVEILLARD J.-Y. et al., 1997 - La pierre de construction en Armorique romaine, L'exemple de Carhaix, Brest, Centre de recherche bretonne et celtique (coll. « Cahiers de Bretagne Occidentale », 17), 121 p., fig.

GALLIEN V., 2009 - École publique, Saint-Georges-de-Montaigu (Vendée), rapport final d'opération, 2 vol., 143 p., fig. (Archives scientifiques du SRA des Pays de la Loire).

GIOT P.-R., 1996 - « L'île Lavret à l'époque romaine », Les dossiers du Centre Régional Archéologique d'Alet, 24, p. 17-37, fig.

GUITTON D. et al., 2005 - L'agglomération antique de Rezé (Loire-Atlantique), le quartier de Saint-Lupien, rapport de fouille programmée annuelle, 118 p., fig. (Archives scientifiques du SRA des Pays de la Loire).

GUITTON D. et al., 2006 - L'agglomération antique de Rezé (Loire-Atlantique), le quartier de Saint-Lupien, fouille programmée 2006-2008, rapport intermédiaire, 2 vol., 43 p., fig. (Archives scientifiques du SRA des Pays de la Loire).

GUYODO J.-N., 2010 - « Approche technologique de l'exploitation minérale », in ARTHUIS R. et al., L'agglomération antique de Rezé (Loire-Atlantique), le quartier Saint-Lupien, fouille programmée 2009, p. 1-6, fig.

LINDEN G., 2004 - Les mots des mines et carrières du Maine-et-Loire, Le Coudray-Macouard, Cheminements, 253 p., fig.

MALIGORNE Y. et al., 2002 - « Extraction et utilisation des granites en Armorique romaine, L'exemple de la carrière de Locuon en Ploërdut (Morbihan) », Gallia, 59, p. 133-143, fig.

MENEZ Y., 1996 - Une ferme de l'Armorique gauloise, Le Boisanne à Plouër-sur-Rance (Côtes-d'Armor), Paris, MSH (coll. « Documents d'Archéologie Française », 58), 267 p., fig.

MONTEIL M. et al., 2009 - L'agglomération antique de Rezé (Loire-Atlantique), le quartier Saint-Lupien, fouille programmée 2006-2008, rapport final 2008, tome III, 105 p., fig. (Archives scientifiques du SRA des Pays de la Loire). 
PERETTI O. DE, 2010 - « Le port antique de Rezé (Loire-Atlantique) », in SERNA V. (dir.), La Loire dessus... dessous, Archéologie d'un fleuve de l'âge du Bronze à nos jours, Dijon, Faton, p. 96-99, fig.

PIRAULT L., 1996 - La Bourderie, Rezé (Loire-Atlantique), DFS de diagnostic archéologique, 13 p., fig.

(Archives scientifiques du SRA des Pays de la Loire).

PIRAULT L., 1997 - La Bourderie sud, Rezé (Loire-Atlantique), DFS de fouille de sauvetage, rapport préliminaire, 9 p., fig. (Archives scientifiques du SRA des Pays de la Loire).

PIRAULT L., 1998 - La Bourderie, Rezé(Loire-Atlantique), fouille programmée, programme pluriannuel 1998-2000, 1re année, 27 p., fig. (Archives scientifiques du SRA des Pays de la Loire).

PIRAULT L., 1999 - La Bourderie, Rezé (Loire-Atlantique), programme pluriannuel 1998-2000, Rapport de fouille programmée, $2 e$ année, 10 p., fig. (Archives scientifiques du SRA des Pays de la Loire). PIRAULT L., 2000 - La Bourderie, Rezé (Loire-Atlantique), programme pluriannuel 1998-2000, rapport de fouille programmée, 3e année, 40 p., fig. (Archives scientifiques du SRA des Pays de la Loire).

PIRAULT L., 2001 - « Recherches récentes sur l'origine de l'urbanisme de la ville antique de Ratiatum (Rezé) », Bulletin de la Société archéologique et historique de Nantes et de Loire-Atlantique, 136, p. 73-88, fig.

PIRAULT L., 2003 - « L'origine antique du parcellaire de la Bourderie à Rezé (44), Fossilisation d'un espace périurbain de l'agglomération gallo-romaine de Ratiatum », Bulletin de la Société archéologique et historique de Nantes et de Loire-Atlantique, 138, p. 45-55, fig.

PIRAULT L., 2004 - « Nantes et Rezé (Loire-Atlantique), développements portuaires et urbains au fond de l'estuaire ", in Approche archéologique de l'environnement et de l'aménagement du territoire ligérien, Actes du colloque de Neuville-aux-Bois, 2002, Orléans, Fédération Archéologique du Loiret, Les Etudes Ligériennes, p. 183-190, fig.

PIRAULT L. et GUITTON D., 2001 - « Rezé, sur les traces de Ratiatum, Loire-Atlantique », Itinéraires du Patrimoine, 254, 40 p., fig.

PIRAULT L. et al., 2001 - «L'atelier de potier de La Bourderie à Rezé (Loire-Atlantique) et sa production ", Revue archéologique de l'Ouest, 18, p. 145-175, fig.

PIRAULT L. et BELLANGER P., 2008 - « L'officine de verrier de la Bourderie à Rezé (Loire-Atlantique) », Bulletin de la Société archéologique et historique de Nantes et de Loire-Atlantique, 143, p. 49-56

POLINSKI A., 2009 - «La pierre sur le site de Saint-Lupien », in ARTHUIS R. et al., L'agglomération antique de Rezé (Loire-Atlantique), le quartier Saint-Lupien, fouille programmée 2006-2008, rapport final 2008, tome II, p. 11-18, fig. (Archives scientifiques du SRA des Pays de la Loire).

\section{NOTES}

1. Nous tenons à remercier J.-C. Bessac (CNRS), M. Monteil et N. Faucherre (université de Nantes) pour leur relecture critique du manuscrit de cet article.

2. C'est le cas notamment de la carrière de l'école publique à Saint-Georges-deMontaigu, en Vendée : Gallien, 2009, p. 28-34, fig.

3. Carte géologique de Nantes au 1/50000, feuille 481 (BRGM).

4. Référence « carte archéologique » du site : $44143075 \mathrm{AH}$.

5. Section AK, parcelle $180 \mathrm{~b}$. 
6. Les productions flaviennes, minoritaires, occupent le comblement sommital.

7. Site $44143004 \mathrm{AH}$.

8. La remontée rapide de la nappe d'eau au fond de la carrière n'a pas permis d'observer correctement la base du comblement. Celle-ci semble néanmoins comporter peu de plaquettes de gneiss et de micaschiste.

9. Site $44143008 \mathrm{AH}$.

10. Au sujet des techniques d'extraction antiques et des outils associés, se reporter à : Adam 2011, p. 23-32, fig.; Bedon 1981, p. 121-137, fig. ; Bedon 1984, p. 101-112, fig. ; Bessac et al., 1996, p. 205-247, fig. ; Bessac 2004, p. 19-25, fig.

11. Nous remercions Ch. Bézier, qui a bien voulu nous faire profiter de son expérience de tailleur de pierre et a examiné cet outil, ainsi que l'Association de sauvegarde du patrimoine historique et artistique de Nozay, qui compte parmi ses membres d'anciens carriers.

12. Nous remercions J.-C. Meuret et D. Trideau, qui ont proposé d'établir un lien entre cet outil et le travail du querneur. Le quernage est l'une des étapes de la chaîne opératoire autrefois en vigueur dans les ardoisières régionales et consistait à débiter, perpendiculairement au plan de schistosité, des blocs ou dalles de schiste en dalles plus petites appelées « repartons » et destinées au fendeur.

\section{RÉSUMÉS}

L'agglomération antique de Rezé a livré une douzaine de sites d'extraction de micaschiste, gneiss et granite pegmatitique. Ces carrières de taille modeste, situées dans les quartiers de la Bourderie et de Saint-Lupien, ont été exploitées pour la plupart dans le courant du $\mathrm{i}^{\mathrm{er}}$ siècle apr. J.-C. Les procédés d'extraction employés semblent laisser une large place à l'utilisation du coin, dont un exemplaire a peut-être été retrouvé sur le site de Saint-Lupien.

About twelve quarries of micaschist, gneiss and pegmatitic granite were found in the ancient small town of Rezé. These small quarries, located in the La Bourderie and Saint-Lupien districts, were exploited in the 1st century AD. The study of quarrying processes reveals that wedges were widely used - one may notably have been discovered on the Saint-Lupien site.

\section{INDEX}

Keywords : Rezé, small town, Gallo-Roman, quarries, quarrying, wedge, construction, gneiss, micaschist

Mots-clés : Rezé, agglomération secondaire, gallo-romain, extraction, construction, gneiss, carrière, micaschiste 


\section{AUTEURS}

\section{ALEXANDRE POLINSKI}

ATER - université de Nantes, UMR 6566 CReAAH, Laboratoire de Recherches archéologiques Chemin de la Censive-du-Tertre - 44313 NANTES Cedex 3.

\section{LIONEL PIRAULT}

Archéologue - Inrap - 4 rue du Tertre - 44470 CARQUEFOU 